\title{
Flight Envelope Protection Control Based on Reference Governor Method in High Angle of Attack Maneuver
}

\author{
Hui Ye, ${ }^{1,2}$ Mou Chen, ${ }^{1,2}$ and Qingxian $W u^{1,2}$ \\ ${ }^{1}$ Jiangsu Key Laboratory of Internet of Things and Control Technologies, Nanjing University of Aeronautics and Astronautics, \\ Nanjing, Jiangsu 210016, China \\ ${ }^{2}$ College of Automation Engineering, Nanjing University of Aeronautics and Astronautics, Nanjing, Jiangsu 210016, China
}

Correspondence should be addressed to Hui Ye; yehuicc@gmail.com

Received 14 September 2014; Revised 17 January 2015; Accepted 21 January 2015

Academic Editor: Giuseppe Rega

Copyright (C) 2015 Hui Ye et al. This is an open access article distributed under the Creative Commons Attribution License, which permits unrestricted use, distribution, and reproduction in any medium, provided the original work is properly cited.

\begin{abstract}
The reference governor strategy is presented for the flight envelope protection control of the aircraft during high angle of attack maneuvers. Limits of key flight parameters and amplitude saturation of control surfaces are explicitly considered by imposing the output and input constraints on the flight system. Firstly, a nominal flight control scheme is designed using eigenstructure assignment approach. The static and dynamic reference governors are discussed for flight envelope protection control of the aircraft during stability-axis roll maneuver. Then, a new reference governor is presented based on step response model. The performance of these reference governors for flight envelope protection is demonstrated and compared by numerical simulations. Results show that the dynamic reference governor and the proposed step response model based reference governor are valid for the flight envelope protection control and the latter is easier to be realized.
\end{abstract}

\section{Introduction}

The control problem for systems with input and output constraints has substantially attracted researchers' attention during the past decades. Various control strategies have been proposed to solve this problem (see [1]). Among these existing strategies, antiwindup is an effective approach to deal with systems with input constraints [2,3], and override can be used to handle output constraints. In addition to this method, there exist other effective techniques to deal with systems with input constraints and output constraints, for example, model predictive control $[4,5]$ or reference governor [6-14].

The reference governor, also called command governor and reference management device, is a practical method to deal with the constrained tracking control problem. It is a nonlinear device which is located between the original reference command and the input to the closed-loop system. The reference governor can modify the original reference in an appropriate way and a virtual command is produced for the closed-loop system to avoid violation of input and output constraints. To establish the inner closed-loop system, a nominal controller should be designed. And the output of the reference governor is employed as the input of the inner closed-loop system. The advantage of this scheme is that the reference governor does not change the performance of inner closed-loop system which is ensured by the nominal controller when the system is far away from its constraints. This is a grateful property for many practical applications which have requirements on both tracking performance and constraint fulfillment.

In the engineering practice, the low-pass filter can be considered as the basic type of the reference governor with openloop form. However, this form is quite conservative. A more effective method is the closed-loop reference governor which can modify the reference based on the current system state. Up to now, several reference governors of this form have been developed. Gilbert et al. [6] studied two discrete-time reference governors including the static and the dynamic forms for linear control systems with state and control constraints. These methodologies were improved in [7], in which the disturbance inputs were considered. A reference governor is proposed based on conceptual tools of predictive control for constrained linear time-invariant systems by Bemporad et al. [8] . 
In [9], it was extended to the time-variant case and obtained a robust command governor. This type of reference governor was applied to an inverted pendulum in [10]. Kogiso and Hirata [11] constructed a reference governor in the form of a piecewise affine function of the state. Reference governors for constrained nonlinear systems have been also studied [1214]. However, when these methods are implemented in the practice of the flight envelope protection control, the admissible sets of the system used in the reference governors are complex and difficult to be obtained.

The flight envelope protection control of the aircraft in high angle of attack maneuver can be treated as a constrained control problem for which the reference governor is available. The safe flight regime is commonly described in terms of constraints on key parameters, airspeed and angle of attack. Other limitations related to the flight state of the aircraft may come from the saturation of control surfaces. The task of the flight envelope protection control is to track the commands from the cockpit. At the same time, the aircraft should stay within the boundaries of the envelope. The traditional limiter scheme is inefficient when the aircraft maneuvers at high angle of attack. Pilots need to monitor cockpit instruments when operating the aircraft to execute the desired maneuvers. Advanced flight envelope protection control system could reduce pilot's workload and improve the safety of the aircraft. Although lots of control laws have been developed for the aircraft to perform maneuvers at high angle of attack well $[15,16]$, few of them take the envelope protection problem into consideration. van Oort et al. [17] developed a controller for F-16/MATV aircraft model to maneuver at high angle of attack combined with a stability-axis roll by a combination of feedback linearization and robust model predictive control (MPC). The constraints on the input and state were transformed to linear matrix inequality (LMI) constraints of the optimization problem of the MPC. In [18], an antiwindup compensator was designed for the aircraft model with input saturation. However, the constraints on outputs were not considered. Falkena et al. [19] investigated the effect of four approaches for the flight envelope protection: control limiting, command limiting, constrained flight control law, and virtual control limiting. The results indicated that for practical implementation command limit is the best choice. But only hard limits were imposed on the commands and the dynamic of the closed-loop system was not considered. In this paper, the reference governor is employed to adjust the original reference based on the current state which contains the dynamic information of the system and guarantees that the system satisfies its input and output constraints during transient or steady.

The principle of the reference governor has similarities with model predictive control $[10,14]$. In absence of information on the future evolution of the reference, two reasonable assumptions can be used to make a prediction: the impulse signal assumption and the step signal assumption. In [6], the static reference governor is based on the impulse signal assumption and other reference governors are based on the step signal assumption. By invoking the latter, it is more convenient to use the increment of the reference signal than the signal itself. Thus, the step response model used in dynamic matrix control (DMC) is more favorable for the design of the reference governor in the practice of flight envelope protection control. Motived by above analysis, the reference governor strategy is presented for the flight envelope protection control of aircraft during the high angle of attack maneuver based on the step signal assumption.

The paper is organized as follows. Section 2 formulates the control problem and basic scheme of the reference governor strategy. Section 3 describes the static and dynamic reference governors proposed by [6], which is designed using the concept of the maximal admissible set. By using the step response model in DMC, a novel reference governor is proposed in Section 4. A constrained aircraft model is introduced in Section 5. Before the reference governor is applied to the flight envelope protection, a nominal flight controller is designed to meet the flying quality requirements using eigenstructure assignment approach. In Section 6, the studied three reference governors are applied to the flight envelope protection of the aircraft in high angle of attack maneuver. The maximal admissible set of the static and dynamic reference governor is calculated while the initial admissible set used in the step response model based reference governor is also given. Simulation results of each reference governor are presented. The advantages and disadvantages of them are discussed. Finally, Section 7 includes some conclusions.

The following notations will be used in this paper. $Z^{+}$ is the set of nonnegative integers. $R^{n}$ is the notation for set of real vectors. The superscript $T$ denotes vector or matrix transpose. The vectors and matrixes used in this paper are notated in boldface and the subsets are notated in decorated letter. $\mathbf{K} \mathscr{P}=\{\mathbf{K x}: \mathbf{x} \in \mathscr{P}\}$ is the image of $\mathscr{P}$ under $\mathbf{K} . \mathbf{f}_{i}$ and $g_{i}$ denote the $i$ th row of the matrix $\mathbf{F}$ and the vector $\mathbf{g}$, respectively.

\section{Problem Formulation and Reference Governor Strategy}

In order to implement the reference governor into the system, the following discrete-time linear time-invariant equation with input and output constraints is considered:

$$
\begin{aligned}
& \mathbf{x}(t+1)=\mathbf{A} \mathbf{x}(t)+\mathbf{B u}(t) \\
& \mathbf{y}(t)=\mathbf{C}_{y} \mathbf{x}(t), \\
& \mathbf{h}(t)=\mathbf{C}_{h} \mathbf{x}(t),
\end{aligned}
$$

where $\mathbf{x} \in R^{n}$ is the state vector and $\mathbf{u} \in R^{m}$ is the input vector which has amplitude saturation constraints; $y \in R^{m}$ is the output which is required to track the commands; $\mathbf{h} \in R^{p}$ is the output on which constraints are imposed; $\mathbf{A}, \mathbf{B}, \mathbf{C}_{y}, \mathbf{C}_{h}$ are matrices with corresponding dimensions. The input and output constraints can be described as $\mathbf{u} \in \mathcal{U}, \mathbf{h} \in \mathscr{H} ; \mathcal{U}$ and $\mathscr{H}$ are convex and compact sets and are usually expressed as the following form:

$$
\begin{aligned}
& \mathscr{U}=\{\mathbf{u}: \underline{\mathbf{u}} \leq \mathbf{u} \leq \overline{\mathbf{u}}, \underline{\mathbf{u}}<0, \overline{\mathbf{u}}>0\}, \\
& \mathscr{H}=\{\mathbf{h}: \underline{\mathbf{h}} \leq \mathbf{h} \leq \overline{\mathbf{h}}, \underline{\mathbf{h}}<0, \overline{\mathbf{h}}>0\} .
\end{aligned}
$$




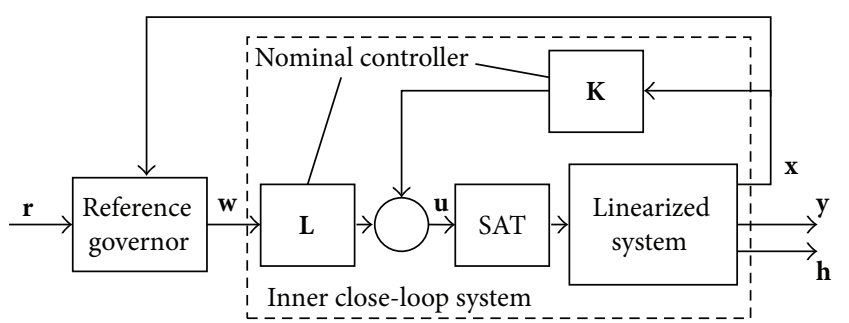

FIGURE 1: Reference governor strategy scheme [6].

The reference governor control strategy consists of a nominal controller and a reference command governor, as depicted in Figure 1. The nominal controller is equipped for obtaining good linear-system properties, that is, the satisfactory tracking performance of $\mathbf{y}$. It is designed via classic linear control methods. Here, a full state feedback control law is given as follows:

$$
\mathbf{u}(t)=\mathbf{K} \mathbf{x}(t)+\mathbf{L w}(t),
$$

where $\mathbf{K}$ is the feedback gain matrix to make system asymptotically stable and $\mathbf{L}$ is the reference input matrix to eliminate the error between $\mathbf{y}$ and $\mathbf{w}$, satisfying

$$
\mathbf{C}_{y}(\mathbf{I}-(\mathbf{A}+\mathbf{B K}))^{-1} \mathbf{B L}=\mathbf{I} .
$$

The virtual command $\mathbf{w}$ is the output of reference governor, which can be considered as a compromise of the original reference $\mathbf{r}$. The task of reference governor is to modify the original reference to avoid constraints violation of input and output and to ensure that the output virtual command is a good approximation of the original reference. The reference governor of closed-loop form can be considered as a prefilter with its parameters varying by the current state and reference.

The object of this paper is rendering the reference governor strategy described above to the flight envelope protection control problem to deal with constraints of key flight parameters and control surfaces.

\section{Static and Dynamic Reference Governor}

In order to address the reference governor theory, the work in [6] is introduced here as a basis of our work. If the nominal controller has been designed, the inner closed-loop system dynamics can be written as

$$
\begin{aligned}
& \mathbf{x}(t+1)=\widetilde{\mathbf{A}} \mathbf{x}(t)+\widetilde{\mathbf{B}} \mathbf{w}(t), \\
& \mathbf{h}(t)=\mathbf{C}_{h} \mathbf{x}(t), \\
& \mathbf{u}(t)=\mathbf{K} \mathbf{x}(t)+\mathbf{L w}(t),
\end{aligned}
$$

where $\widetilde{\mathbf{A}}=\mathbf{A}+\mathbf{B K}, \widetilde{\mathbf{B}}=\mathbf{B L}$.

3.1. Static Reference Governor. Since the information of future behavior of $\mathbf{r}$ is unknown, it is needed to assume that the future $\mathbf{r}$ is of a certain shape when we make a decision on the admissible $\mathbf{r}$ at current time [6]. A reasonable assumption is

$$
\mathbf{r}(\tau)=\mathbf{0}, \quad \text { for } \tau>t
$$

Under this assumption, the evolution of system (5) is similar to a zero-input response. We just need to find an invariant subset of the constrained set and render the state of the system within the subset. This leads to a static reference governor design methodology as follows.

According to [6], the static reference governor is given by

$$
\mathbf{w}(t)=\boldsymbol{\sigma}(\mathbf{r}(t), \mathbf{x}(t)) \mathbf{r}(t),
$$

where $\boldsymbol{\sigma}=\operatorname{diag}\left(\sigma_{1}, \sigma_{2}, \ldots, \sigma_{m}\right)$ is a diagonal matrix and each parameter is a nonlinear function of the reference $\mathbf{r}(t)$ and the current state of the system $\mathbf{x}(t)$.

For the discrete-time system (1), $\mathscr{U}$ and $\mathscr{H}$ are C-sets. The control law (3) is designed to guarantee that $\mathbf{A}+\mathbf{B K}$ is Hurwitz stable. Assume that $\mathscr{P}$ is an invariant C-set in $R^{n}$ for the system (5) when $\mathbf{w}=\mathbf{0}$ and $\mathbf{K} \mathscr{P} \subseteq \mathscr{U}, \mathbf{C}_{h} \mathscr{P} \subseteq \mathscr{H}$. One can design the reference governor as

$$
\begin{aligned}
& \boldsymbol{\sigma}(\mathbf{r}(t), \mathbf{x}(t)) \\
& =\underset{\boldsymbol{\sigma}}{\operatorname{argmin}}\left\{\|\mathbf{r}-\mathbf{w}\|: \sigma_{i} \in[0,1], i=1, \ldots, m,\right. \\
& \widetilde{\mathbf{A}} \mathbf{x}+\widetilde{\mathbf{B}} \boldsymbol{\sigma} \mathbf{r} \in \mathscr{P}, \mathbf{K} \mathbf{x}+\mathbf{L} \boldsymbol{\sigma} \mathbf{r} \in \mathcal{U}\} .
\end{aligned}
$$

The reference governor in (7) can guarantee that for all $\mathbf{x}(0) \epsilon$ $\mathscr{P}$ and for all $\mathbf{r}(t)$ the constraints are not violated [20]. Here $\|\cdot\|$ is an arbitrary norm on $R^{m}$. Since a norm can be considered as a particular measure of the distance, the optimization is to make $\mathbf{w}$ as close to $\mathbf{r}$. In this work, 1-norm is selected that we can treat the optimization problem as a linear programming (LP) problem. LP software can be used to calculate the result quickly. Other gauge functions can be defined to approximate the original reference in a more proper manner which is not discussed here.

The invariant set $\mathscr{P}$ is limited by the input constraint set $\mathcal{U}$ and the control gain $\mathbf{K}$. If a high gain feedback control law is used in the nominal controller, it will result in an extremely small $\mathscr{P}$ which is impracticable in engineering applications. This is a serious defect of the static reference governor which will be illustrated in simulation.

3.2. Dynamic Reference Governor. Another reasonable strategy to predict the future behavior of the reference is to assume that it remains invariant from time $t$ onwards. Reviewing [810] yields

$$
\mathbf{r}(\tau)=\mathbf{r}(t) \text { for } \tau>t
$$

Most of the existing reference governors are based on this assumption, such as the dynamic reference governor. It is constructed as a first-order filter with its parameter $\boldsymbol{\sigma}$ depending on the reference $\mathbf{r}(t)$, the state of the filter $\mathbf{w}(t)$, and the state of the system $\mathbf{x}(t)$. The filter is designed as

$$
\mathbf{w}(t+1)=\mathbf{w}(t)+\boldsymbol{\sigma}(\mathbf{r}(t), \mathbf{w}(t), \mathbf{x}(t))(\mathbf{r}(t)-\mathbf{w}(t)) .
$$


Using $\overline{\mathbf{x}}(t)$ to denote the augmented state vector $\left[\mathbf{w}^{T}(t), \mathbf{x}^{T}(t)\right]^{T}$, the augmented system can be written as follows:

$$
\begin{gathered}
\overline{\mathbf{x}}(t+1)=\overline{\mathbf{A}} \overline{\mathbf{x}}(t)+\overline{\mathbf{B}} \boldsymbol{\sigma}(\mathbf{r}(t), \overline{\mathbf{x}}(t))(\mathbf{r}(t)-\mathbf{w}(t)), \\
\mathbf{u}(t)=\overline{\mathbf{K}} \overline{\mathbf{x}} \in \mathscr{U}, \\
\mathbf{h}(t)=\overline{\mathbf{C}} \overline{\mathbf{x}} \in \mathscr{H},
\end{gathered}
$$

where $\overline{\mathbf{A}}=\left[\begin{array}{ll}\mathbf{I} & 0 \\ \widetilde{\mathbf{B}} & \widetilde{\mathbf{A}}\end{array}\right], \overline{\mathbf{B}}=\left[\begin{array}{l}\mathbf{I} \\ \mathbf{0}\end{array}\right], \overline{\mathbf{C}}=\left[\begin{array}{ll}\mathbf{0} & \mathbf{C}_{h}\end{array}\right], \overline{\mathbf{K}}=\left[\begin{array}{ll}\mathbf{L} & \mathbf{K}\end{array}\right]$.

Similar to the static reference governor, the dynamic reference governor can be designed by using the following method.

For augmented system (11), $\overline{\mathbf{A}}$ is Schur stable. Assume that $\overline{\mathscr{P}}$ is an invariant C-set in $R^{n+m}$ for the system when $\mathbf{r}=\mathbf{w}$ and $\overline{\mathbf{K}} \overline{\mathscr{P}} \subseteq \mathscr{U}, \overline{\mathbf{C}} \overline{\mathscr{P}} \subseteq \mathscr{H}$. Then, the dynamic reference governor can be designed as

$$
\begin{aligned}
& \boldsymbol{\sigma}(\mathbf{r}(t), \overline{\mathbf{x}}(t)) \\
& =\underset{\boldsymbol{\sigma}}{\operatorname{argmin}}\left\{\|\mathbf{w}+\boldsymbol{\sigma}(\mathbf{r}-\mathbf{w})-\mathbf{r}\|: \sigma_{i} \in[0,1], i=1, \ldots, m,\right. \\
& \quad \overline{\mathbf{A}} \overline{\mathbf{x}}+\overline{\mathbf{B}} \boldsymbol{\sigma}(\mathbf{r}-\mathbf{w}) \in \overline{\mathscr{P}}\} .
\end{aligned}
$$

The reference governor in (11) can guarantee that for all $\overline{\mathbf{x}}(0) \in \overline{\mathscr{P}}$ and for all $\mathbf{r}(t)$ the constraints are not violated.

3.3. Calculation of the Maximal Admissible Set. Although $\mathscr{P}$ or $\overline{\mathscr{P}}$ is an arbitrary C-set limited by $\mathscr{U}$ and $\mathscr{H}$, the excessively conservative selection of the set will lead to a small feasible solution of $\boldsymbol{\sigma}$, which means the distance between $\mathbf{r}$ and $\mathbf{w}$ is relatively large. The best selection of $\mathscr{P}$ or $\overline{\mathscr{P}}$ is the maximal admissible set of the inner closed-loop system, which can be defined by

$$
\begin{gathered}
\mathscr{X}^{\infty}=\left\{\mathbf{x}: \mathbf{C}_{h} \widetilde{\mathbf{A}}^{t} \mathbf{x} \in \mathscr{H}, \mathbf{K} \widetilde{\mathbf{A}}^{t} \mathbf{x} \in \mathscr{U}, t \in Z^{+}\right\}, \text {for static; } \\
\overline{\mathscr{X}}^{\infty}=\left\{\overline{\mathbf{x}}: \overline{\mathbf{C A}}^{t} \overline{\mathbf{x}} \in \mathscr{H}, \overline{\mathbf{K A}}^{t} \mathbf{x} \in \mathcal{U}, t \in Z^{+}\right\}, \text {for dynamic. }
\end{gathered}
$$

Obviously, the maximal admissible set is an invariant set and satisfies the conditions of the reference governor. Because $\mathscr{H}$ and $\mathcal{U}$ have been assumed to have the polyhedral forms, $\mathscr{X}^{\infty}$ and $\bar{X}^{\infty}$ are also polyhedral sets. A numerical method developed to calculate the polyhedral invariant set given in [21] can be used to obtain the maximal admissible sets. Take the static case as an example and the maximal admissible set in dynamic reference governor can be obtained by the same procedures which are described as follows.

Procedure. Define the initial admissible set $\mathscr{X}^{0}$ with $\mathbf{w}=\mathbf{0}$ as

$$
\begin{aligned}
\mathscr{X}^{0} & =\left\{\mathbf{x}: \mathbf{C}_{h} \mathbf{x} \in \mathscr{H}, \mathbf{K} \mathbf{x} \in \mathscr{U}\right\} \\
& =\left\{\mathbf{x}: \underline{\mathbf{h}} \leq \mathbf{C}_{h} \mathbf{x} \leq \overline{\mathbf{h}}, \underline{\mathbf{u}} \leq \mathbf{K} \mathbf{x} \leq \overline{\mathbf{u}}\right\} .
\end{aligned}
$$

Let $\mathbf{F}^{0}=\left[\mathbf{C}_{h}^{T},-\mathbf{C}_{h}^{T}, \mathbf{K}^{T},-\mathbf{K}^{T}\right]^{T}$ and $\mathbf{g}^{0}=\left[\overline{\mathbf{h}}^{T},-\underline{\mathbf{h}}^{T}, \overline{\mathbf{u}}^{T},-\underline{\mathbf{u}}^{T}\right]^{T}$. The constraints representation for $\mathscr{X}^{0}$ is expressed as

$$
\mathscr{X}^{0}=\left\{\mathbf{x}: \mathbf{F}^{0} \mathbf{x} \leq \mathbf{g}^{0}\right\}=\left\{\mathbf{x}: \mathbf{f}_{i}^{0} \mathbf{x} \leq g_{i}^{0}\right\} \text {. }
$$

Step 1. Set the initial polyhedron $\mathscr{X}^{0}$ and give a tolerance $\varepsilon>$ 0 . The maximal number of steps is set as $k_{\max }$. Furthermore, the initial number is $k=0$.

Step 2. Compute the preimage set of the set $\mathscr{X}^{k}$ which is denoted by $\mathscr{P} \mathscr{R}\left(\mathscr{X}^{k}\right)$ and given by

$$
\mathscr{P} \mathscr{R}\left(\mathscr{X}^{k}\right)=\left\{\mathbf{x}: \mathbf{F}^{k} \mathbf{A} \mathbf{x} \leq \mathbf{g}^{k}\right\}
$$

Step 3. Set $\mathscr{X}^{k+1}=\mathscr{X}^{k} \cap \mathscr{P} \mathscr{R}\left(\mathscr{X}^{k}\right)$ and let the matrices $\mathbf{F}^{k+1}$ and $\mathbf{g}^{k+1}$ be those associated with the constraints representation of the set $\mathscr{X}^{k+1}$. Namely, $\mathscr{X}^{k+1}=\left\{\mathbf{x}: \mathbf{F}^{k+1} \mathbf{x} \leq\right.$ $\left.\mathbf{g}^{k+1}\right\}$.

Step 4. If $\mathscr{X}^{k} \subseteq(1+\varepsilon) \mathscr{X}^{k+1}$, set $\mathscr{X}^{\infty}=\mathscr{X}^{k}$ and stop successfully. If $k>k_{\max }$, stop indeterminately. Otherwise, set $k=k+1$ and go to Step 2 .

It can be proved that the maximal admissible set can be finitely determined when the system is asymptotically stable [20]. The inner closed-loop system is asymptotically stable due to the nominal control law. Thus, $\mathscr{X}^{\infty}$ in the static reference governor can be obtained easily. However, the augmented system matrix $\overline{\mathbf{A}}$ is just Schur stable. It is not assured that the maximal admissible set of the augmented system can be finitely determined. In real computation, we always use proper $\varepsilon$ or $k_{\max }$ to obtain an approximate result. Even so, the calculation is usually time-consuming and the resulting set is complex which will be shown in the application of the flight envelope protection.

3.4. Calculation of the State Uncertainty Set. For a practical aircraft system, it is usually not possible or economically feasible to measure all the states accurately. For example, the angles of attack and sideslip are difficult to measure accurately in the aircraft control system due to the existence of external disturbance. However, an observer or Kalman filter can be employed to provide corresponding estimates of them. We divide the state vector $\mathbf{x}(t)$ into two parts: $\mathbf{x}_{1}(t) \in R^{n_{1}}$ denotes the states which can be measured accurately, and $\mathbf{x}_{2}(t) \in R^{n_{2}}$ denotes the unmeasured states. The unmeasured states can be estimated as follows:

$$
\mathbf{z}(t)=\mathbf{x}_{2}(t)+\boldsymbol{\xi}(t)
$$

where $\mathbf{z}(t)$ is the estimate of the actual $\mathbf{x}_{2}(t)$ and $\boldsymbol{\xi}(t)$ is a bounded noise satisfying $\boldsymbol{\xi}(t) \in \mathscr{D} \subset R^{n_{2}}$. Here $\mathscr{D}$ is a polyhedral set with $\mathbf{0} \in \mathscr{D}$ which can be expressed as $\mathscr{D}=$ $\left\{\boldsymbol{\xi}: \mathbf{F}_{d} \boldsymbol{\xi}<\mathbf{g}_{d}\right\}$.

In such case, a state uncertainty set of $\mathbf{x}_{2}(t)$ should be obtained before the reference governor is designed. The method in [22] is employed here to calculate the exact state 
uncertainty set. The set of unmeasured states depending on the estimate value $\mathbf{z}(t)$ can be written as

$$
\mathscr{X}_{z}(t)=\left\{\mathbf{x}_{2}:-\mathbf{F}_{d} \mathbf{x}_{2}<\mathbf{g}_{d}-\mathbf{F}_{d} \mathbf{z}(t)\right\}
$$

The exact state uncertainty set $\mathscr{X}_{\Sigma}(t \mid t)$ is calculated using the following set-valued recursions [22]:

$$
\begin{gathered}
\mathscr{X}_{\Sigma}(t \mid t)=\mathscr{X}_{\Sigma}(t \mid t-1) \cap \mathscr{X}_{z}(t), \\
\mathscr{X}_{\Sigma}(t+1 \mid t)=\left\{\widetilde{\mathbf{A}}_{1} \mathbf{x}_{1}(t)\right\}+\widetilde{\mathbf{A}}_{2} \mathscr{X}_{\Sigma}(t \mid t)+\{\widetilde{\mathbf{B}} \mathbf{w}(t)\},
\end{gathered}
$$

where $\widetilde{\mathbf{A}}_{1}, \widetilde{\mathbf{A}}_{2}$ are partitioned matrices of $\widetilde{\mathbf{A}}$ and $\{\cdot\}$ denotes a singleton. Assume that $\mathscr{X}_{\Sigma}(t \mid t-1)$ has the polyhedral form, such as $\mathscr{X}_{\Sigma}(t \mid t-1)=\left\{\mathbf{x}_{2}: \mathbf{F}_{\Sigma}^{t} \mathbf{x}_{2}<\mathbf{g}_{\Sigma}^{t}\right\}$, and $\mathbf{x}_{2}(t)=\mathbf{C}_{z} \mathbf{x}(t)$. Then, the recursive procedures can be expressed as follows:

$$
\begin{aligned}
& \mathscr{X}_{\Sigma}(t \mid t) \\
& =\left\{\mathbf{x}_{2}: \overline{\mathbf{F}}_{\Sigma}^{t} \mathbf{x}_{2}<\overline{\mathbf{g}}_{\Sigma}^{t}, \overline{\mathbf{F}}_{\Sigma}^{t}=\left[\begin{array}{c}
\mathbf{F}_{\Sigma}^{t} \\
-\mathbf{F}_{d}
\end{array}\right], \overline{\mathbf{g}}_{\Sigma}^{t}=\left[\begin{array}{c}
\mathbf{g}_{\Sigma}^{t} \\
\mathbf{g}_{d}-\mathbf{F}_{d} \mathbf{z}(t)
\end{array}\right]\right\}, \\
& \mathscr{X}_{\Sigma}(t+1 \mid t) \\
& =\left\{\mathbf{x}_{2}: \mathbf{F}_{\Sigma}^{t+1} \mathbf{x}_{2}<\mathbf{g}_{\Sigma}^{t+1}, \mathbf{F}_{\Sigma}^{t+1}=\overline{\mathbf{F}}_{\Sigma}^{t}\left(\mathbf{C}_{z} \widetilde{\mathbf{A}}_{2}\right)^{-1},\right. \\
& \left.\quad \mathbf{g}_{\Sigma}^{t+1}=\overline{\mathbf{g}}_{\Sigma}^{t}+\overline{\mathbf{F}}_{\Sigma}^{t}\left(\mathbf{C}_{z} \widetilde{\mathbf{A}}_{2}\right)^{-1} \mathbf{C}_{z}\left(\widetilde{\mathbf{A}}_{1} \mathbf{x}_{1}(t)+\widetilde{\mathbf{B}} \mathbf{w}(t)\right)\right\} .
\end{aligned}
$$

In the design of the reference governor, $\mathscr{X}_{\Sigma}(t \mid t)$ is used by modifying (8) and (12) to (21) and (22), respectively. Then, we have

$$
\begin{aligned}
& \boldsymbol{\sigma}(\mathbf{r}(t), \mathbf{x}(t)) \\
& =\underset{\boldsymbol{\sigma}}{\operatorname{argmin}}\left\{\|\mathbf{r}-\mathbf{w}\|: \sigma_{i} \in[0,1], i=1, \ldots, m,\right. \\
& \widetilde{\mathbf{A}}\left[\begin{array}{l}
\mathbf{x}_{1} \\
\widehat{\mathbf{x}}_{2}
\end{array}\right]+\widetilde{\mathbf{B}} \boldsymbol{\sigma} \mathbf{r} \in \mathscr{P}, \\
& \left.\mathbf{K}\left[\begin{array}{l}
\mathbf{x}_{1} \\
\widehat{\mathbf{x}}_{2}
\end{array}\right]+\mathbf{L} \boldsymbol{\sigma} \mathbf{r} \in \mathscr{U}, \forall \widehat{\mathbf{x}}_{2} \in \mathscr{X}_{\Sigma}(t \mid t)\right\},
\end{aligned}
$$

$$
\begin{aligned}
\boldsymbol{\sigma}(\mathbf{r}(t), \overline{\mathbf{x}}(t)) & \underset{\boldsymbol{\sigma}}{\operatorname{argmin}}\left\{\|\mathbf{w}+\boldsymbol{\sigma}(\mathbf{r}-\mathbf{w})-\mathbf{r}\|: \sigma_{i} \in[0,1], i=1, \ldots, m,\right. \\
& \left.\overline{\mathbf{A}}\left[\begin{array}{c}
\mathbf{w} \\
\mathbf{x}_{1} \\
\widehat{\mathbf{x}}_{2}
\end{array}\right]+\overline{\mathbf{B}} \boldsymbol{\sigma}(\mathbf{r}-\mathbf{w}) \in \overline{\mathscr{P}}, \forall \widehat{\mathbf{x}}_{2} \in \mathscr{X}_{\Sigma}(t \mid t)\right\} .
\end{aligned}
$$

According to (21) and (22), we can obtain a static reference governor and a dynamic reference governor with state uncertainty sets.

\section{Reference Governor Based on Step Response Model}

In this section, a new reference governor is proposed based on the methodology introduced in Section 3. The constant reference assumption can also be expressed using the increment of reference signal at next time which is

$$
\Delta \mathbf{r}(\tau)=\mathbf{0} \quad \text { for } \tau>t
$$

Since the increment signal is used, the step response model is employed to describe the relationship between the increment input and the output of the inner closed-loop system. It will be seen that the design of the reference governor is greatly simplified based on this model. Firstly, the step response model of the system should be deduced from the state-space representation (5) by the similar method used in DMC [23]. In order to obtain the step response model, we need the following assumptions.

Assumption 1. The increments of the reference become zero after the sampling time $t$; that is, $\Delta \mathbf{w}(t+1)=\Delta \mathbf{w}(t+2)=$ $\cdots=\mathbf{0}$.

Assumption 2. The settling time of the system is shorter than $N$ sample interval.

From Assumption 2, we know that the inner closed-loop system will achieve steady state from $t+N-1$ onwards. The states of the system will be constant, as $\mathbf{x}(t+N-1)=\mathbf{x}(t+N)=$ $\mathbf{x}(t+N+1)=\cdots$. In general, the nominal controller can ensure the stability of the inner closed-loop system. Thus, this assumption is reasonable.

Consider the $N$-steps response of system (5) when $\mathbf{w}$ keeps constant; that is, $\Delta \mathbf{w}(t)=\mathbf{0}$. Then, the following plant is obtained:

$$
\mathbf{X}(t+1)=\mathbf{M}_{s s} \mathbf{X}(t),
$$

where $\mathbf{X}(t)=\left[\mathbf{x}^{T}(t), \mathbf{x}^{T}(t+1), \ldots, \mathbf{x}^{T}(t+N-1)\right]^{T}, \mathbf{X}(t+1)=$ $\left[\mathbf{x}^{T}(t+1), \mathbf{x}^{T}(t+2), \ldots, \mathbf{x}^{T}(t+N)\right]^{T}$, and

$$
\mathbf{M}_{s s}=\left[\begin{array}{cccccc}
\mathbf{0} & \mathbf{I}_{n \times n} & \mathbf{0} & \cdots & \mathbf{0} & \mathbf{0} \\
\mathbf{0} & \mathbf{0} & \mathbf{I}_{n \times n} & \cdots & \mathbf{0} & \mathbf{0} \\
\vdots & \vdots & \vdots & \ddots & \vdots & \vdots \\
\mathbf{0} & \mathbf{0} & \mathbf{0} & \cdots & \mathbf{0} & \mathbf{I}_{n \times n} \\
\mathbf{0} & \mathbf{0} & \mathbf{0} & \cdots & \mathbf{0} & \mathbf{I}_{n \times n}
\end{array}\right]_{N \times N}
$$

Next, consider the $N$-steps response of the system (5) with a step signal input under the zero initial condition. Namely, there are $\mathbf{x}(t)=\mathbf{0}, \mathbf{w}(t-1)=\mathbf{0}, \Delta \mathbf{w}(t)=\mathbf{w}(t)=\mathbf{w}(t+1)=\cdots$ and

$$
\begin{aligned}
\mathbf{x}(t+1) & =\widetilde{\mathbf{A}} \mathbf{x}(t)+\widetilde{\mathbf{B}} \mathbf{w}(t)=\widetilde{\mathbf{B}} \Delta \mathbf{w}(t), \\
\mathbf{x}(t+2) & =\widetilde{\mathbf{A}} \mathbf{x}(t+1)+\widetilde{\mathbf{B}} \mathbf{w}(t+1), \\
& =\widetilde{\mathbf{A}} \widetilde{\mathbf{B}} \Delta \mathbf{w}(t)+\widetilde{\mathbf{B}} \Delta \mathbf{w}(t)=(\widetilde{\mathbf{A}} \widetilde{\mathbf{B}}+\widetilde{\mathbf{B}}) \Delta \mathbf{w}(t),
\end{aligned}
$$




$$
\begin{aligned}
\mathbf{x}(t+3) & =\widetilde{\mathbf{A}} \mathbf{x}(t+2)+\widetilde{\mathbf{B}} \mathbf{w}(t+2) \\
& =\widetilde{\mathbf{A}}(\widetilde{\mathbf{A}} \widetilde{\mathbf{B}}+\widetilde{\mathbf{B}}) \Delta \mathbf{w}(t)+\widetilde{\mathbf{B}} \Delta \mathbf{w}(t) \\
& =\left(\widetilde{\mathbf{A}}^{2} \widetilde{\mathbf{B}}+\widetilde{\mathbf{A}} \widetilde{\mathbf{B}}+\widetilde{\mathbf{B}}\right) \Delta \mathbf{w}(t) \\
& \vdots \\
\mathbf{x}(t+N) & =\left(\widetilde{\mathbf{A}}^{N-1} \widetilde{\mathbf{B}}+\widetilde{\mathbf{A}}^{N-2} \widetilde{\mathbf{B}}+\cdots+\widetilde{\mathbf{A}} \widetilde{\mathbf{B}}+\widetilde{\mathbf{B}}\right) \Delta \mathbf{w}(t) .
\end{aligned}
$$

The above equations can be simplified as

$$
\mathbf{X}(t+1)=\mathbf{S} \Delta \mathbf{w}(t),
$$

where

$$
\mathbf{S}=\left[\begin{array}{c}
\widetilde{\mathbf{B}} \\
\widetilde{\mathbf{A}} \widetilde{\mathbf{B}}+\widetilde{\mathbf{B}} \\
\vdots \\
\widetilde{\mathbf{A}}^{N-1} \widetilde{\mathbf{B}}+\widetilde{\mathbf{A}}^{N-2} \widetilde{\mathbf{B}}+\cdots \widetilde{\mathbf{A}} \widetilde{\mathbf{B}}+\widetilde{\mathbf{B}}
\end{array}\right]_{N \times m} .
$$

Because of the additivity property of the linear system, the response of the system to any increment of the input under nonzero initial conditions can be written as

$$
\begin{gathered}
\mathbf{X}(t+1)=\mathbf{M}_{s s} \mathbf{X}(t)+\mathbf{S} \Delta \mathbf{w}(t), \\
\mathbf{H}(t)=\mathbf{C}_{s s} \mathbf{X}(t), \\
\mathbf{U}(t)=\mathbf{K}_{s s} \mathbf{X}(t)+\mathbf{L}_{s s} \Delta \mathbf{w}(t)+\mathbf{L}_{s s} \mathbf{w}(t-1),
\end{gathered}
$$

where $\mathbf{H}(t)=\left[\mathbf{h}^{T}(t), \mathbf{h}^{T}(t+1), \ldots, \mathbf{h}^{T}(t+N-1)\right]^{T}, \mathbf{U}(t)=$ $\left[\mathbf{u}^{T}(t), \mathbf{u}^{T}(t+1), \ldots, \mathbf{u}^{T}(t+N-1)\right]^{T}$, and

$$
\begin{gathered}
\mathbf{C}_{s s}=\left[\begin{array}{llll}
\mathbf{C}_{h} & & & \\
& \mathbf{C}_{h} & & \\
& & \ddots & \\
& & & \mathbf{C}_{h}
\end{array}\right], \\
\mathbf{K}_{s s}=\left[\begin{array}{cccc}
\mathbf{K} & & & \\
& \mathbf{K} & & \\
& & \ddots & \\
& & \mathbf{K}
\end{array}\right], \quad \\
\end{gathered}
$$

Now, we employ the principle used in Section 3 to the step response model (29). We can obtain that $\mathbf{M}_{s s}$ is Schur stable. On the basis of this conclusion, the maximal admissible set of the system when $\Delta \mathbf{w}(t)=0$ can be found. The initial admissible set $\mathscr{X}^{0}$ of system (5) with a known w can be written as

$$
\mathscr{X}^{0}(\mathbf{w})=\left\{\mathbf{x}: \mathbf{C}_{h} \mathbf{x} \in \mathscr{H}, \mathbf{K} \mathbf{x}+\mathbf{L w} \in \mathscr{U}\right\} .
$$

Since $\mathbf{w}(t-1)$ is known at time $t$, it is obvious that the maximal admissible set of system (29) with $\Delta \mathbf{w}(t)=0$ is given by

$$
\mathscr{X}_{s s}=\left\{\mathbf{X}: \mathbf{x}(t+i) \in \mathscr{X}^{0}(\mathbf{w}(t-1)), i=0, \ldots, N-1\right\} .
$$

Using the related results of reference governor in Section 3, we can design the reference governor as

$$
\Delta \mathbf{w}(t)=\boldsymbol{\sigma}(\Delta \mathbf{r}(t), \mathbf{X}(t)) \Delta \mathbf{r}(t),
$$

where $\Delta \mathbf{r}(t)$ denotes the increment of the original reference. The parameter $\boldsymbol{\sigma}$ is calculated via the following optimization problem:

$$
\begin{gathered}
\boldsymbol{\sigma}(\Delta \mathbf{r}(t), \mathbf{X}(t)) \\
=\underset{\boldsymbol{\sigma}}{\operatorname{argmin}}\left\{\|\Delta \mathbf{r}(t)-\Delta \mathbf{w}(t)\|: \sigma_{i} \in[0,1], i=1, \ldots, m,\right. \\
\left.\mathbf{M}_{s s} \mathbf{X}(t)+\mathbf{S} \boldsymbol{\sigma} \Delta \mathbf{r}(t) \in \mathscr{X}_{s s}\right\} .
\end{gathered}
$$

Compared with the dynamic reference governor, a significant advantage of this reference governor is that the maximal admissible set (32) is explicit and is easy to obtain. Although the complexity of optimization computation is increased due to the use of an $N$-steps prediction model (29), the prediction step number $N$ is finite. Another noticeable feature of this reference governor is that it is designed without using the measurement of states. A virtual step response model is used to generate the $\mathbf{X}(t)$ signal instead. So the state uncertainty set is not considered here.

\section{Nominal Controller Design for the Flight Envelope Protection}

In this section, a constrained aircraft model is introduced. A nominal controller is designed for the aircraft model to embody desirable flying qualities properties.

5.1. Constrained Aircraft Model in High Angle of Attack Maneuver. The stability-axis roll maneuver is a classic maneuver at high angle of attack. During this maneuver, the altitude and velocity can be seen as a constant and the angle of attack is maintained at a high value near the edge of the envelope. At the same time, the sideslip angle is limited to zero. A linear aircraft model can be obtained from [16]. The trim point is selected so that the linear aircraft model can efficiently approximate the nonlinear dynamics during the high angle of attack maneuver. The trim conditions are $V_{0}=70 \mathrm{~m} / \mathrm{s}, \alpha_{0}=$ $15.6474^{\circ}, \delta_{h 0}=0.5851^{\circ}$, and $H_{0}=1000 \mathrm{~m}$. Since the candidate aircraft does not equip the thrust vector control device, the maneuver is performed by using the conventional aerodynamic control surface. During the maneuver, the engine thrust is set to maximum value to provide adequate power. Thus, we do not consider thrust as a control input of the system. Three controlled variables are selected to be related to cockpit control according to the three channels of the aircraft. The linearized mode is

$$
\begin{gathered}
\dot{\mathbf{x}}^{\prime}(t)=\mathbf{A}_{\text {trim }} \mathbf{x}^{\prime}(t)+\mathbf{B}_{\text {trim }} \mathbf{u}(t), \\
\mathbf{y}(t)=\mathbf{C}_{\text {trim }} \mathbf{x}^{\prime}(t) .
\end{gathered}
$$

In (35), the state vector $\mathbf{x}^{\prime}=(u, v, w, p, q, r)^{T}$ consists of the body components of linear velocity and angular velocity; the 


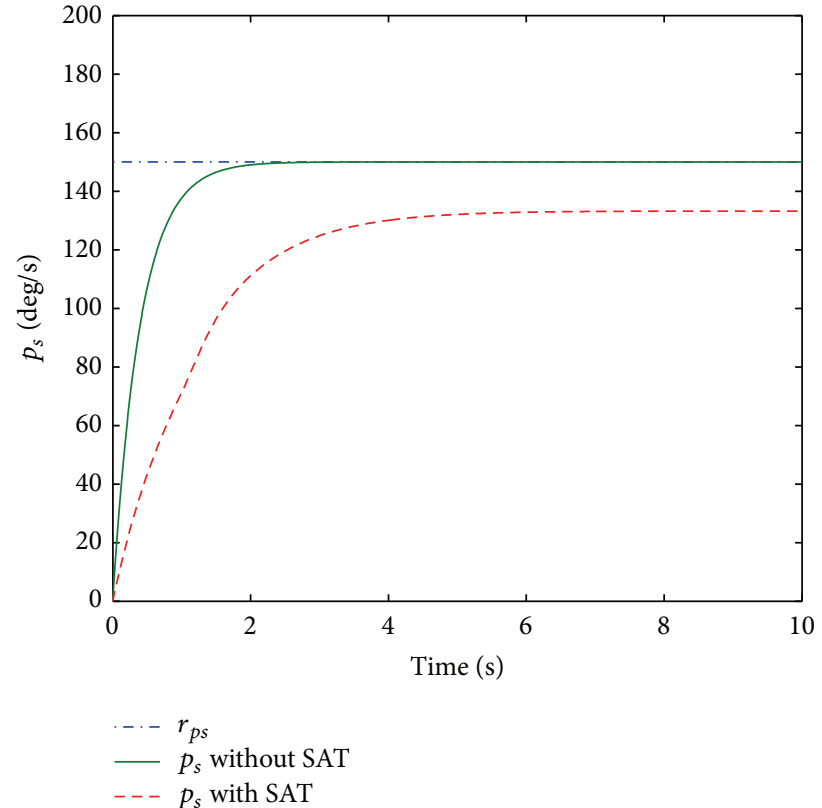

(a)

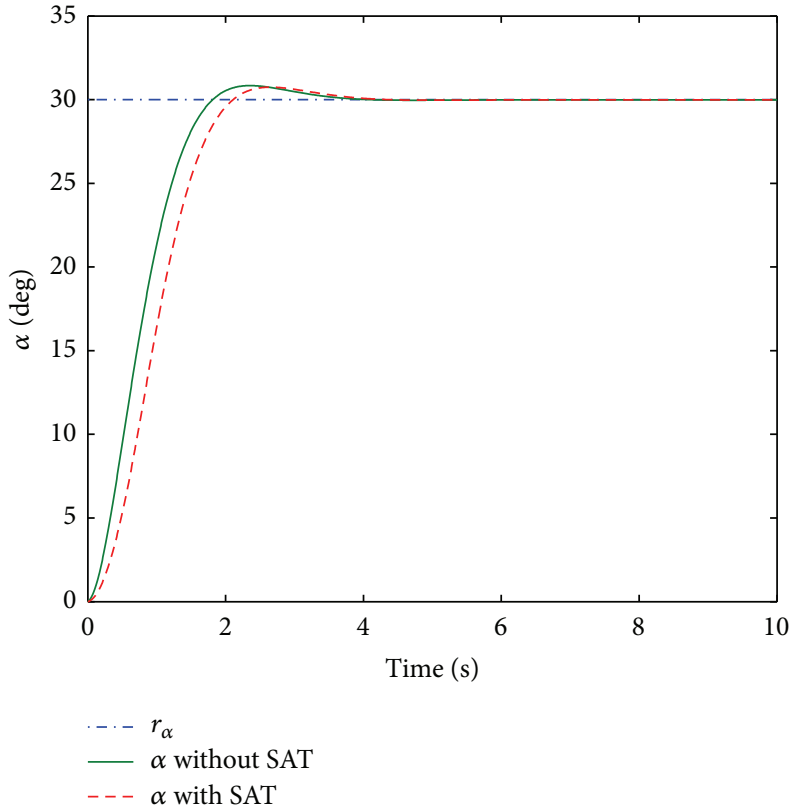

(b)

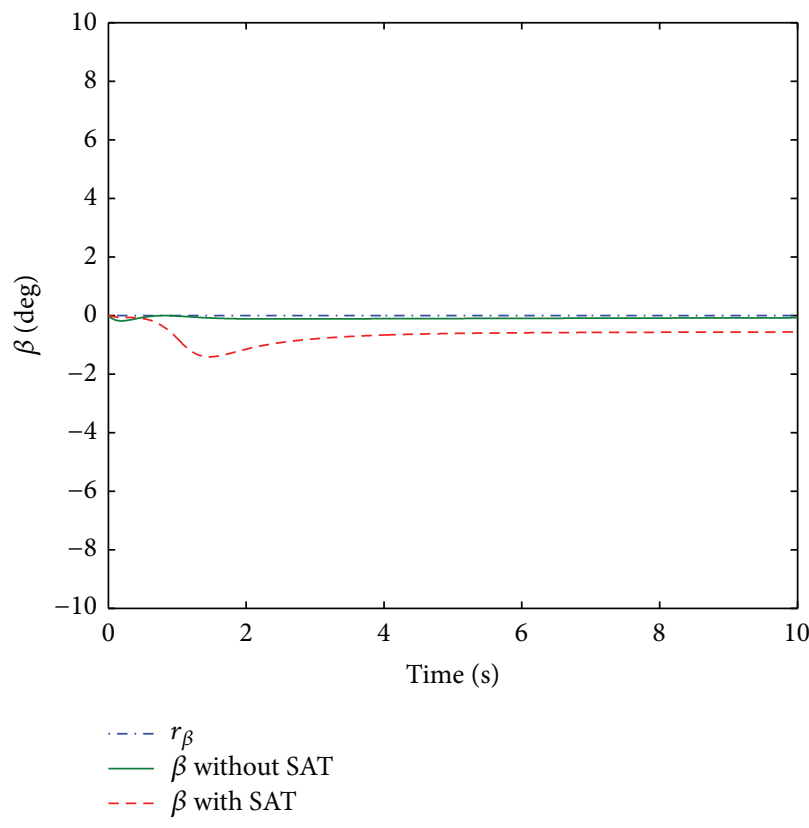

(c)

FIGURE 2: Simulation results of the nominal controller.

inputs vector $\mathbf{u}=\left(\delta_{h}, \delta_{a}, \delta_{r}\right)^{T}$ includes deflections of the horizontal stabilator, aileron, and rudder; the performance output vector is $\mathbf{y}=\left(p_{s}, \alpha, \beta\right)^{T}$, in which $\alpha$ and $\beta$ are angles of attack and sideslip, respectively. $p_{s}$ is the stability-axis roll rate defined as $p_{s}=p \cos \alpha+r \sin \alpha . \mathbf{A}_{\text {trim }}, \mathbf{B}_{\text {trim }}, \mathbf{C}_{\text {trim }}$ are system matrices derived by linearization at the trim point.

For high angle of attack maneuvers, the body-axis rates $(p, q, r)^{T}$ are usually substituted by stability-axis rates $\left(p_{s}, q_{s}, r_{s}\right)^{T}$. We also use $(V, \alpha, \beta)^{T}$ to describe the velocity vector rather than the body components $(u, v, w)^{T}$. A transformation is introduced to rewrite model (35) with a new state vector $\mathbf{x}=\left(V, \alpha, \beta, p_{s}, q_{s}, r_{s}\right)^{T}$. The new system matrices are as follows:

$$
\begin{aligned}
\mathbf{A} & =\mathbf{T A}_{\text {trim }} \mathbf{T}^{-1} \\
& =\left[\begin{array}{cccccc}
-0.0529 & -0.0608 & 0.0035 & 0 & -0.0184 & 0 \\
-0.2174 & -0.3906 & 0.0047 & 0 & 0.9142 & 0 \\
0 & 0 & -0.1221 & 0.0038 & 0 & -0.9934 \\
0 & 0 & -12.2528 & -1.0333 & 0.0010 & 1.0455 \\
0.0050 & -0.6269 & -0.0140 & -0.0008 & -0.5592 & -0.0028 \\
0 & 0 & 4.5806 & 0.1937 & 0.0023 & -0.5195
\end{array}\right],
\end{aligned}
$$




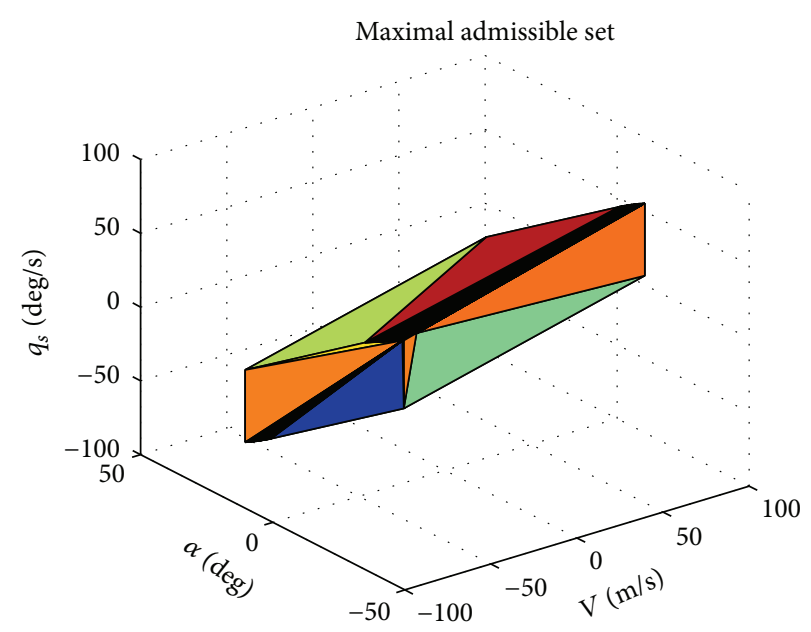

(a)

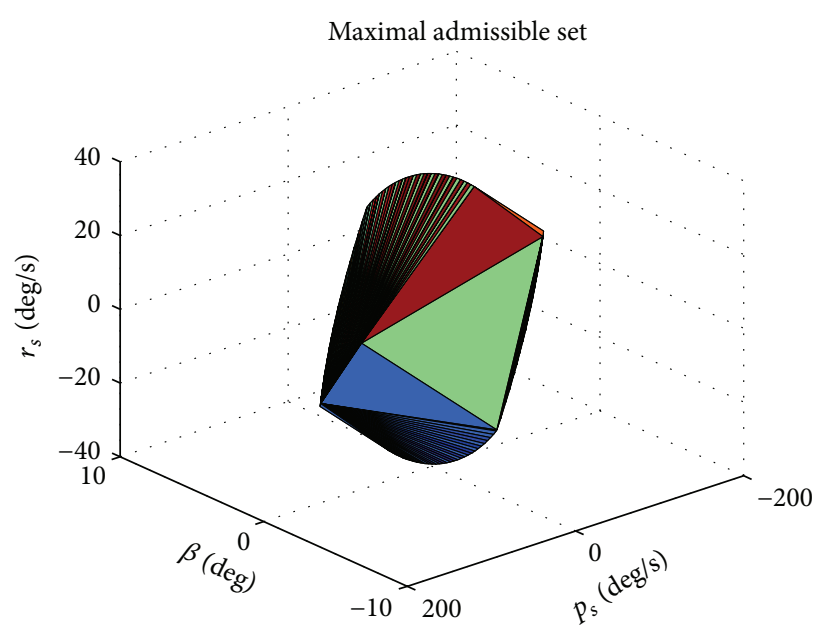

(b)

Figure 3: The maximal admissible set of the static reference governor.

$$
\begin{gathered}
\mathbf{B}=\mathbf{T B}_{\text {trim }}=\left[\begin{array}{ccc}
-0.0330 & 0 & 0 \\
-0.0339 & 0 & 0 \\
0 & 0.0073 & 0.0189 \\
0 & -7.1671 & 1.0921 \\
-2.0397 & 0 & 0 \\
0 & 1.7516 & -1.0080
\end{array}\right], \\
\mathbf{C}_{y}=\mathbf{C}_{\text {trim }} \mathbf{T}^{-1}=\left[\begin{array}{llllll}
0 & 0 & 0 & 1 & 0 & 0 \\
0 & 1 & 0 & 0 & 0 & 0 \\
0 & 0 & 1 & 0 & 0 & 0
\end{array}\right],
\end{gathered}
$$

where

$$
\mathbf{T}=\left[\begin{array}{cccccc}
0.9629 & 0 & 0.2697 & 0 & 0 & 0 \\
-0.2208 & 0 & 0.7882 & 0 & 0 & 0 \\
0 & 0.8185 & 0 & 0 & 0 & 0 \\
0 & 0 & 0 & 55.1724 & 0 & 15.4536 \\
0 & 0 & 0 & 0 & 57.2958 & 0 \\
0 & 0 & 0 & -15.4536 & 0 & 55.1724
\end{array}\right]
$$

is the transition matrix.

During the stability-axis roll maneuver, the velocity $V$, angle of attack $\alpha$, and the stability-axis roll rate $p_{s}$ should be limited to prevent the aircraft from the state out of control. We assume that the symmetric constraints are imposed on these key parameters. Besides, boundaries of other state variables are chosen for simplified calculation and data availability. The dynamics of the actuators are neglected and only amplitude saturations are considered. Then, the input and output constraints are written as

$$
\mathcal{U}=\left\{\begin{aligned}
&-25^{\circ} \leq \delta_{h} \leq 25^{\circ} \\
& \mathbf{u}: \quad-20^{\circ} \leq \delta_{a} \leq 20^{\circ} \\
&-30^{\circ} \leq \delta_{r} \leq 30^{\circ}
\end{aligned}\right\},
$$

$$
\mathscr{H}=\left\{\begin{aligned}
-70 \mathrm{~m} / \mathrm{s} & \leq V \leq 70 \mathrm{~m} / \mathrm{s} \\
-30^{\circ} & \leq \alpha \leq 30^{\circ} \\
-30^{\circ} & \leq \beta \leq 30^{\circ} \\
\mathbf{h}: \quad 150^{\circ} / \mathrm{s} & \leq p_{s} \leq 150^{\circ} / \mathrm{s} \\
-150^{\circ} / \mathrm{s} & \leq q_{s} \leq 150^{\circ} / \mathrm{s} \\
-150^{\circ} / \mathrm{s} & \leq r_{s} \leq 150^{\circ} / \mathrm{s}
\end{aligned}\right\},
$$

and $\mathbf{h}=\mathbf{x}, \mathbf{C}_{h}=\mathbf{I}$.

5.2. Nominal Controller Design. The nominal controller is designed to make the performance of the closed-loop system satisfy the flying quality specifications described in MILSTD-1797A [24]. Among the existing approaches, the eigenstructure assignment developed in [25] is an effective method and widely used in flight control design. An advantage of this approach is that it leads to an inner closed-loop system of loworder which is important for the calculation of invariant set. It will be seen that the complexity of the computation and the resulting maximal admissible set grows exponentially by the order of the closed-loop system.

The performance output variables $\left(p_{s}, \alpha, \beta\right)^{T}$ are considered as the controlled variables corresponding to the roll, pitch, and yaw axis of aircraft, respectively. They are expected to have the desired response characteristics. The desired closed-loop eigenstructure consists of the desired modes for the closed-loop aircraft system, along with the eigenvectors associated with these modes. Firstly, we select a set of desired closed-loop eigenvalues $\lambda^{d}$ to meet the requirements of closed-loop frequency and damping of the various dynamic modes proposed in MIL-STD-1797A. Equation (39) illustrates the candidate eigenvalues $\lambda^{d}$ along with desired eigenvectors. The longitudinal short period mode has an eigenvalue pair of $-1.5 \pm 1.3229 i$, and the Dutch roll mode also has an eigenvalue pair of $-3.09 \pm 2.7251 i$. For roll mode, a single negative real eigenvalue -2.5 is given. And then, we assign a matching eigenvector for each eigenvalue, as indicated in $V^{d}$. 

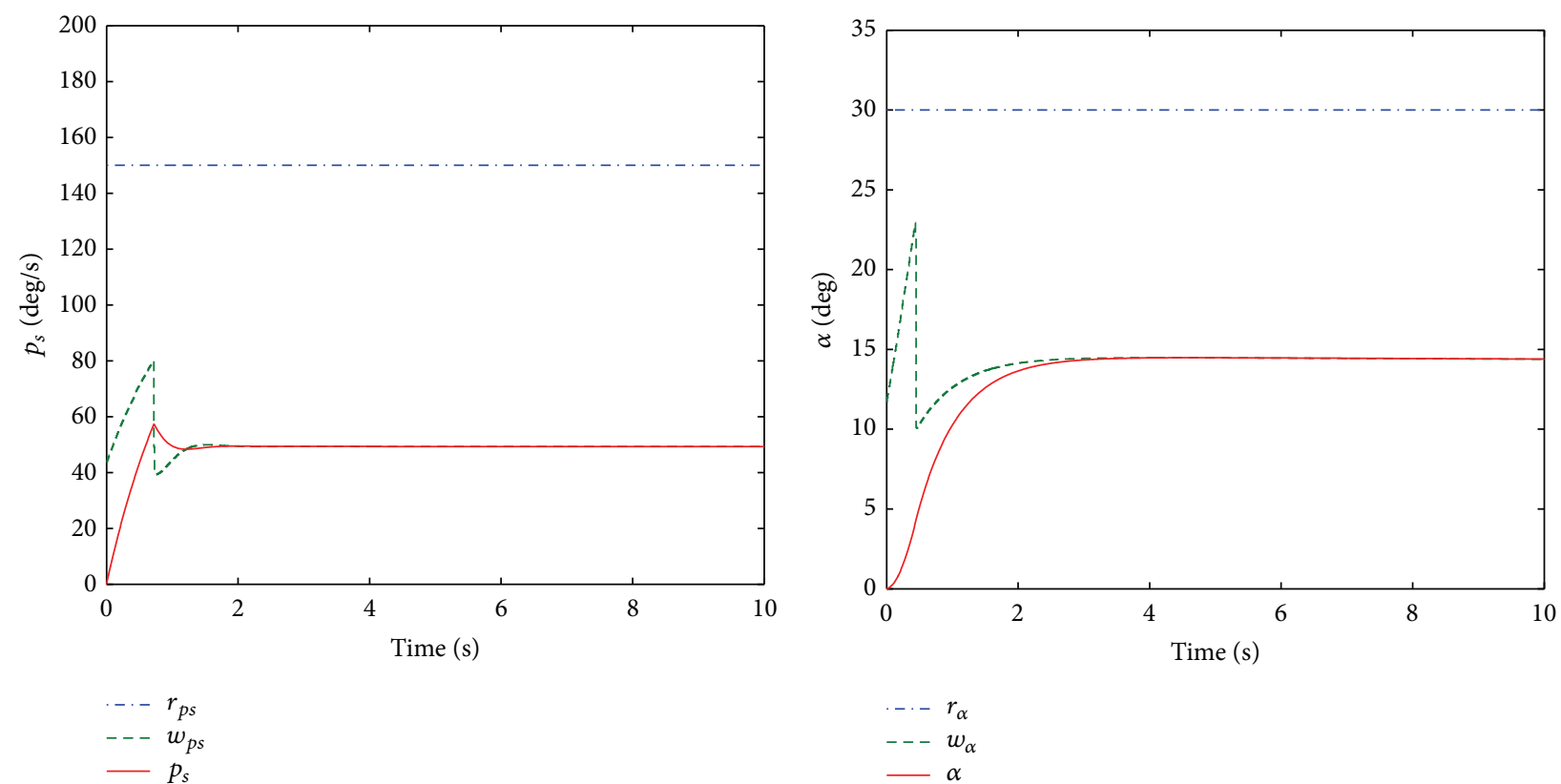

(a)
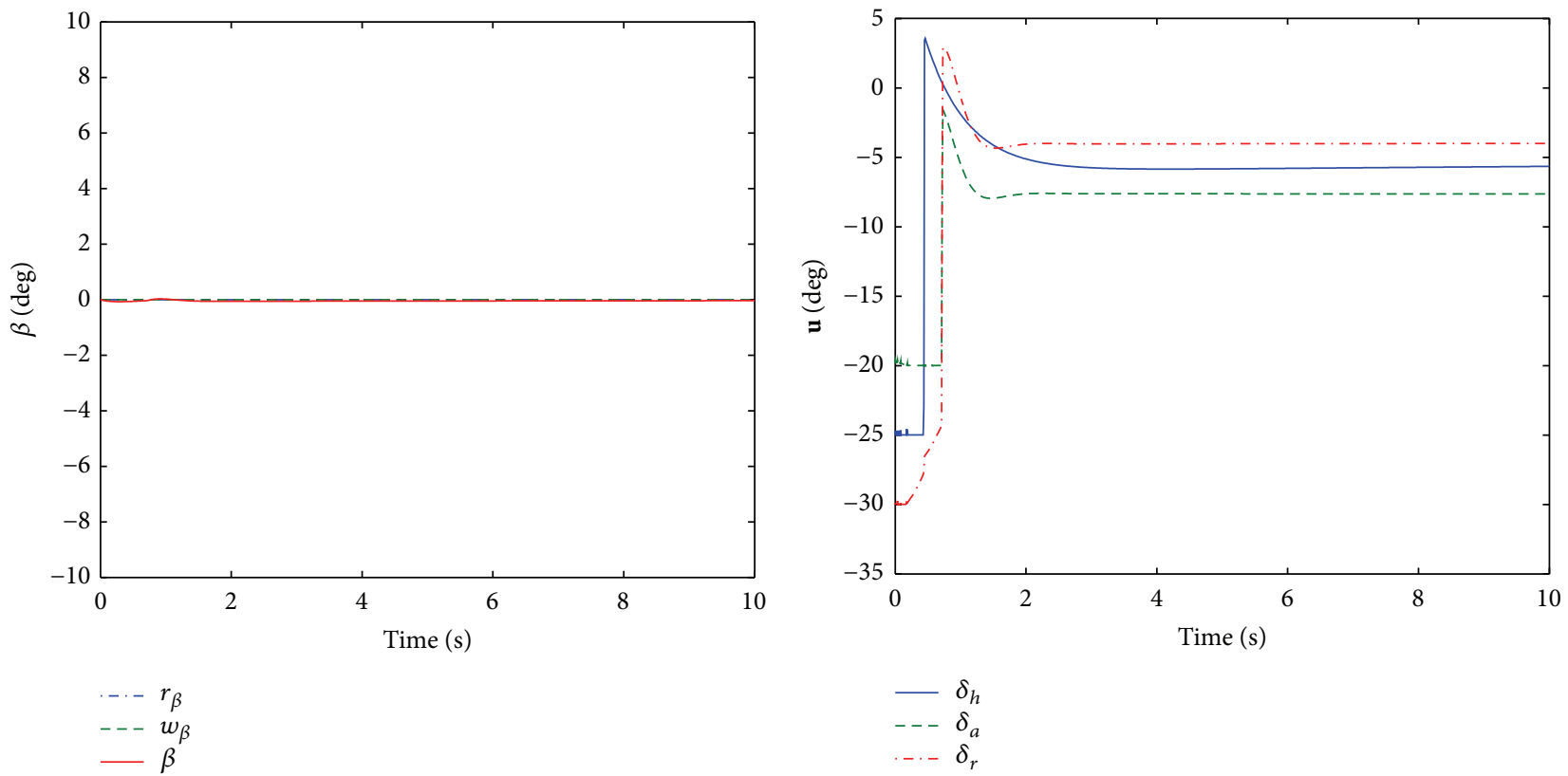

(c)

(d)

FIGURE 4: Simulation results of the static reference governor.

Here " $x$ " denotes an entry that is unconstrained which can be selected arbitrarily. Furthermore, all other values denote hard eigenvector constraints (" 1 " denotes related and "0" denotes unrelated):

$$
\begin{aligned}
\lambda^{d} & =[\underbrace{-1.5+1.3229 i-1.5-1.3229 i}_{\text {short period }} \\
V^{d} & =\left[\begin{array}{ccccc}
\times & \times & \times & \times & \text { dutch roll } \\
1 & \times & 0 & 0 & 0 \\
0 & 0 & 1 & \times & 0 \\
0 & 0 & 0 & 0 & 1 \\
\times & 1 & 0 & 0 & 0 \\
0 & 0 & \times & 1 & 0
\end{array}\right] \begin{array}{c}
-3.09+2.7251 i-3.09-2.7251 i \\
p_{s} \\
r_{s}
\end{array}
\end{aligned}
$$




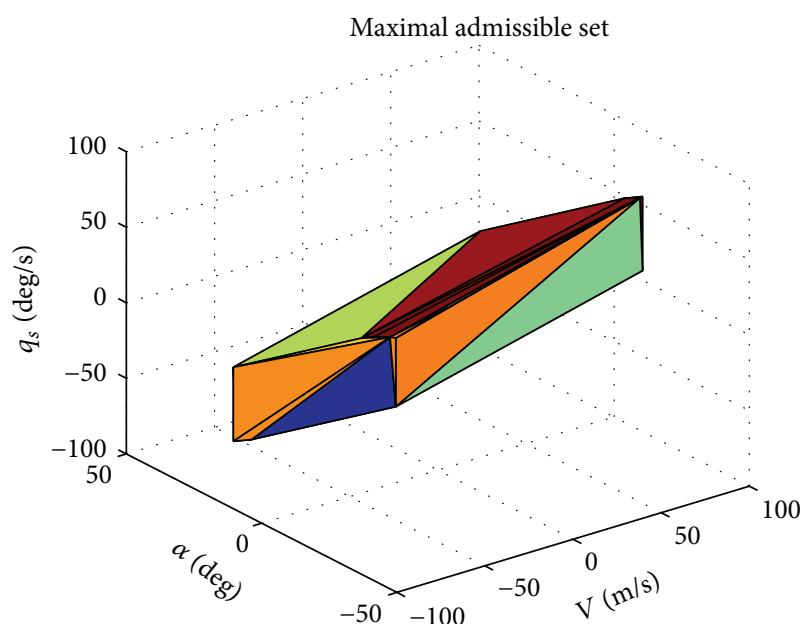

(a)

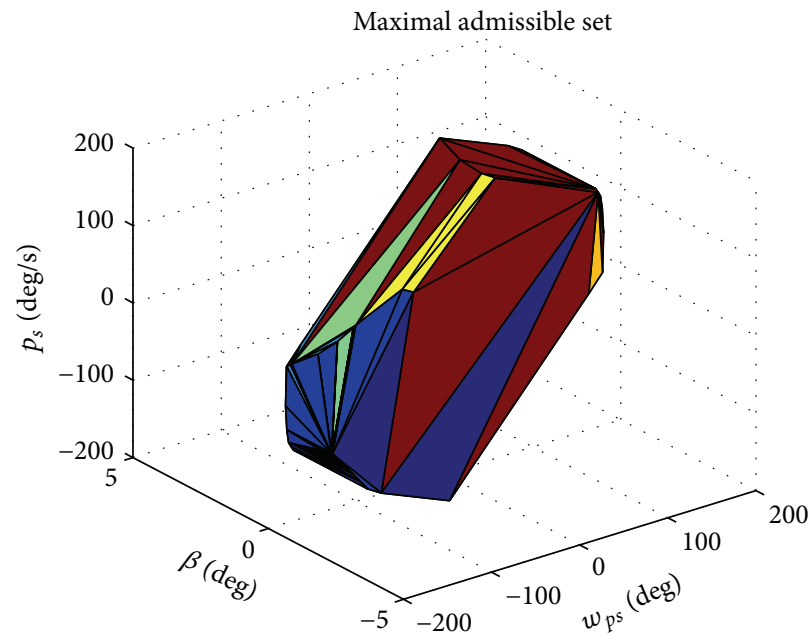

(c)

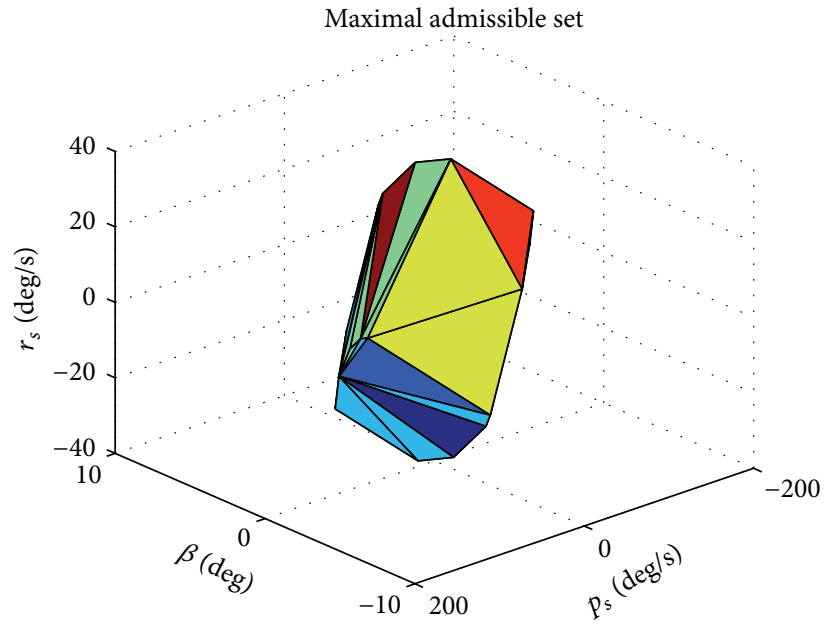

(b)

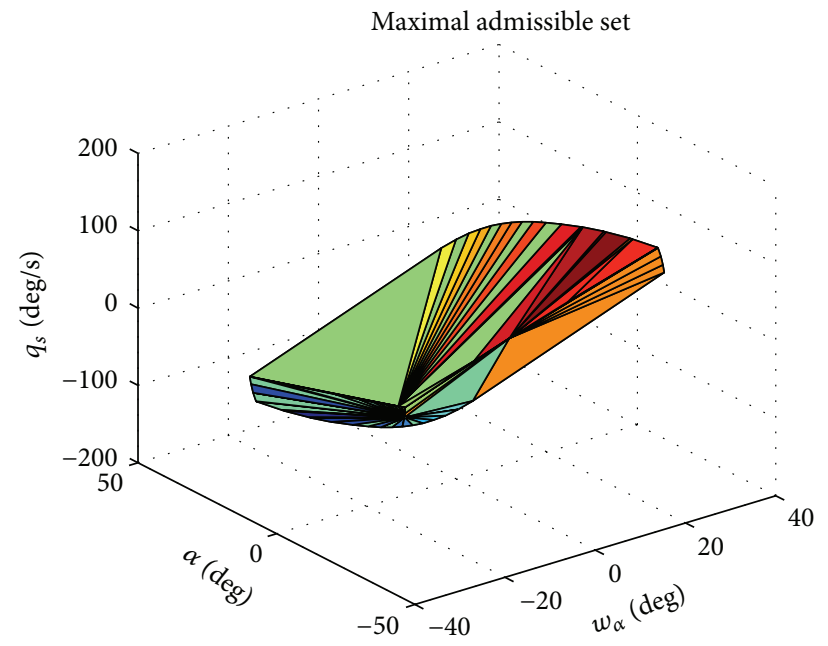

(d)

FIGURE 5: The maximal admissible set of the dynamic reference governor.

Assuming that all states of the plant are measurable, the state feedback control law is used to assign the desired eigenstructure; that is, $\mathbf{u}=\mathbf{K x}$. The system matrix $\mathbf{A}$ is 6-dimensional but only 5 eigenvalues have been given. Although eigenvalues $\lambda_{i}$ can be assigned arbitrarily via state feedback for a controllable system, the eigenvectors only can be assigned when they are on the subspace spanned by $\left(\lambda_{i} \mathbf{I}-\mathbf{A}\right)^{-1} \mathbf{B}$. On the other hand, the velocity $V$ is a long-term variable in the state vector $\mathbf{x}$. The last eigenvalue and eigenvector are relevant to the long-term modes of the system and should be selected to eliminate effects of $V$ on the short-term modes. When the last eigenvalue is fixed to -0.0554 , the corresponding eigenvector is approximated to $(1,0,0,0,0,0)^{T}$, and the elements related to $V$ in the other eigenvectors are almost equal to zero. Then, the effects of long-term mode are removed.

Finally, the reference input matrix $\mathbf{L}$ is designed to eliminate the steady state error between reference commands and responses of the system. Under this case, (4) should be satisfied. As a result, a state feedback control law is obtained as $\mathbf{u}=\mathbf{K} \mathbf{x}+\mathbf{L w}$, in which

$$
\begin{gathered}
\mathbf{K}=\left[\begin{array}{cccccc}
-0.2880 & 1.3102 & -0.0040 & -0.0004 & 0.9892 & -0.0019 \\
-0.0156 & 0.0006 & -4.7689 & 0.2917 & 0.0011 & 1.2520 \\
-0.1027 & 0.0037 & -20.0769 & 0.5712 & 0.0062 & 7.2590
\end{array}\right], \\
\mathbf{L}=\left[\begin{array}{cccc}
0.0006 & -2.1306 & 0.0055 \\
-0.4972 & -0.0009 & 6.4892 \\
-0.9736 & -0.0059 & 27.3191
\end{array}\right] .
\end{gathered}
$$

5.3. Simulation Results of the Nominal Controller. The obtained nominal control law was demonstrated by the simulation of MATLAB in which a high angle of attack stabilityaxis roll maneuver is performed. Extreme references of a step angle of attack command $r_{\alpha}$ with 30-degree amplitude are examined. At the same time, a step stability-axis roll rate command $r_{p s}$ with an amplitude of 150 degrees per second is 


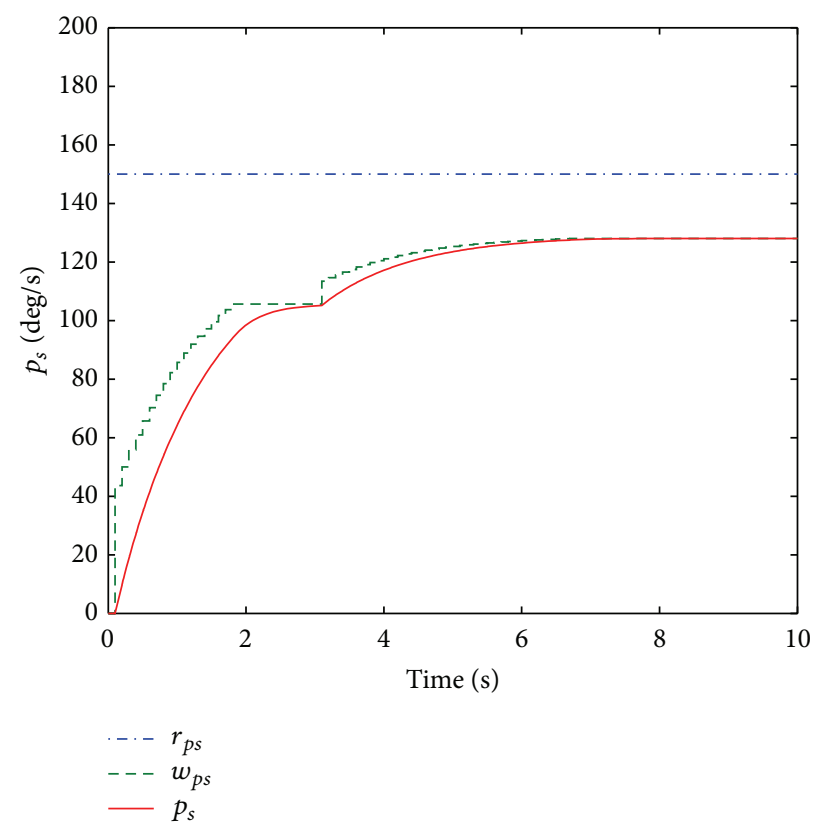

(a)

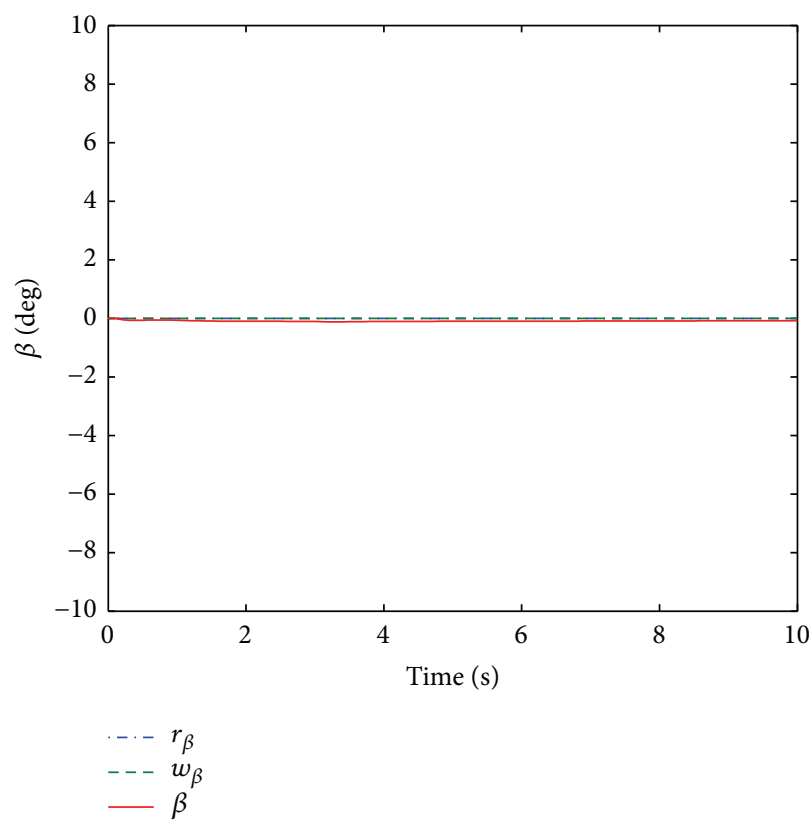

(c)

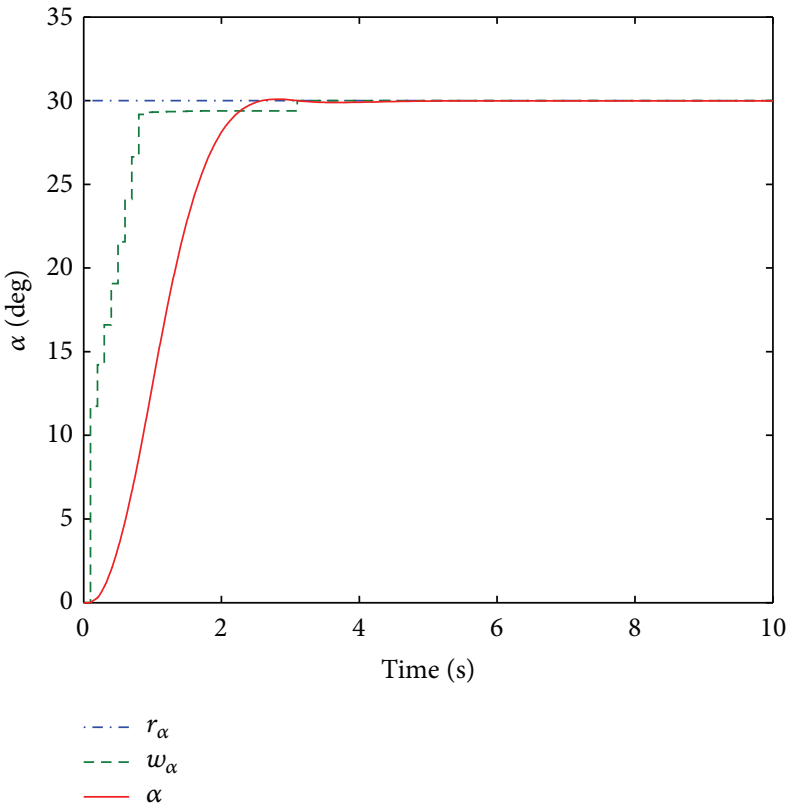

(b)

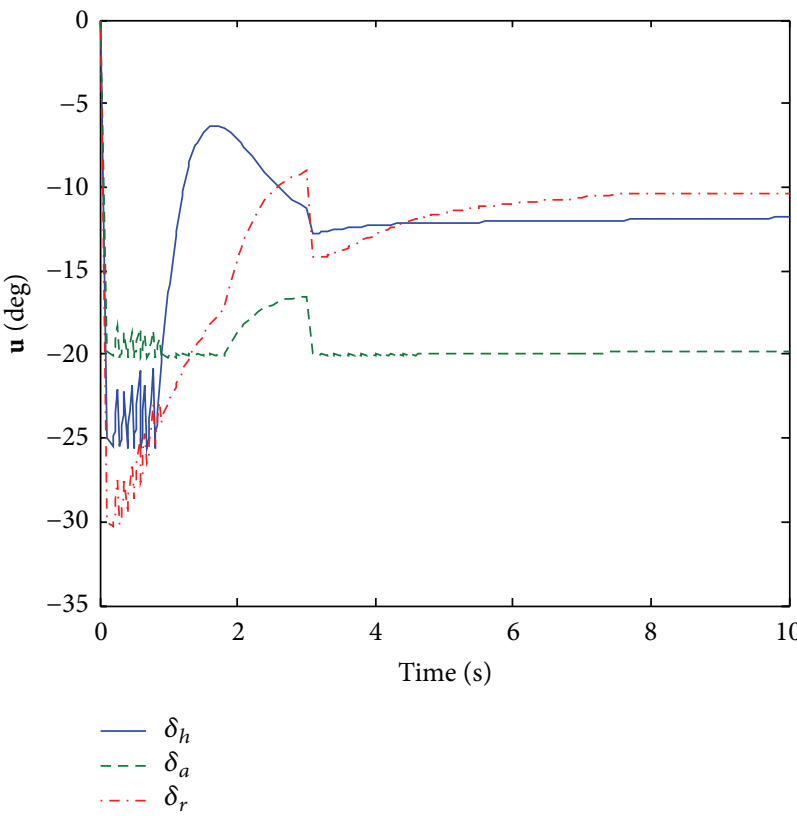

(d)

FiguRE 6: Simulation results of the dynamic reference governor.

given. The sideslip angle $r_{\beta}$ remains zero during the maneuver. Figure 2 includes the time plots of the simulation results, in which two cases are shown: model with actuator saturations (dashed line) and without actuator saturations (solid line). The solid lines in the figure show that good tracking performance is achieved for $p_{s}, \alpha$, and $\beta$ in the absence of actuator saturation. When saturations are imposed, the steady state of $p_{s}$ cannot reach its command due to the limit on aileron, and the nonlinear characteristic of the saturations will result in an error of $\beta$. On the other hand, the response of $\alpha$ has overshoot which is out of the limit on the angle of attack.
Therefore, a reference governor is needed to deal with the input and output constraints.

\section{Simulation Study of Reference Governor for Flight Envelope Protection}

Based on the previous analysis, the nominal control is insufficient to cope with the input and output constraints. The reference governor is essential for the flight envelope protection during the high angle of attack maneuver. 


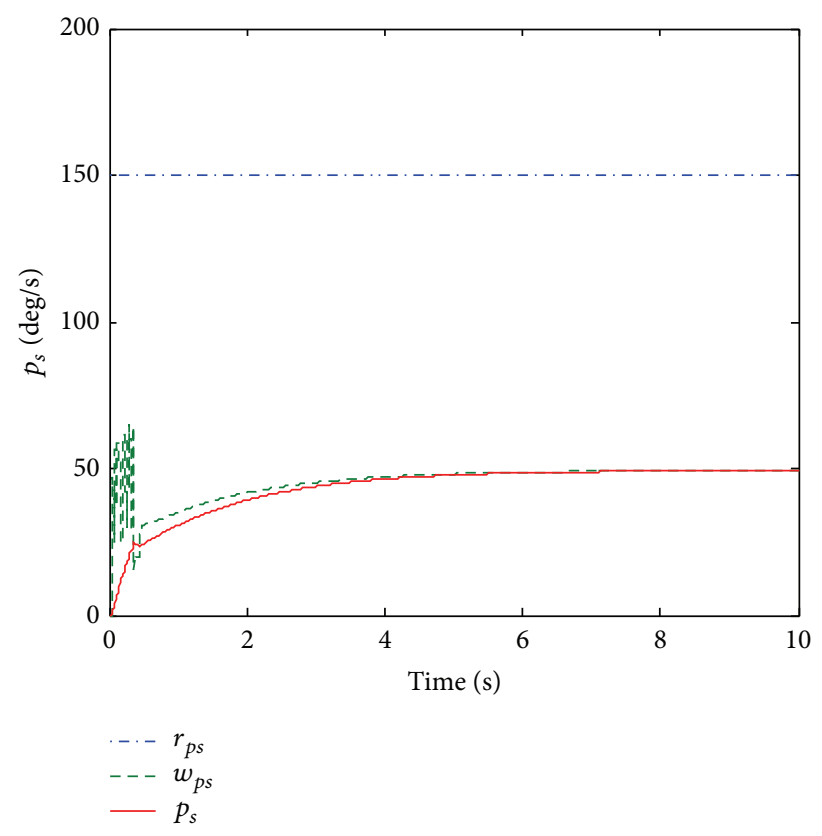

(a)

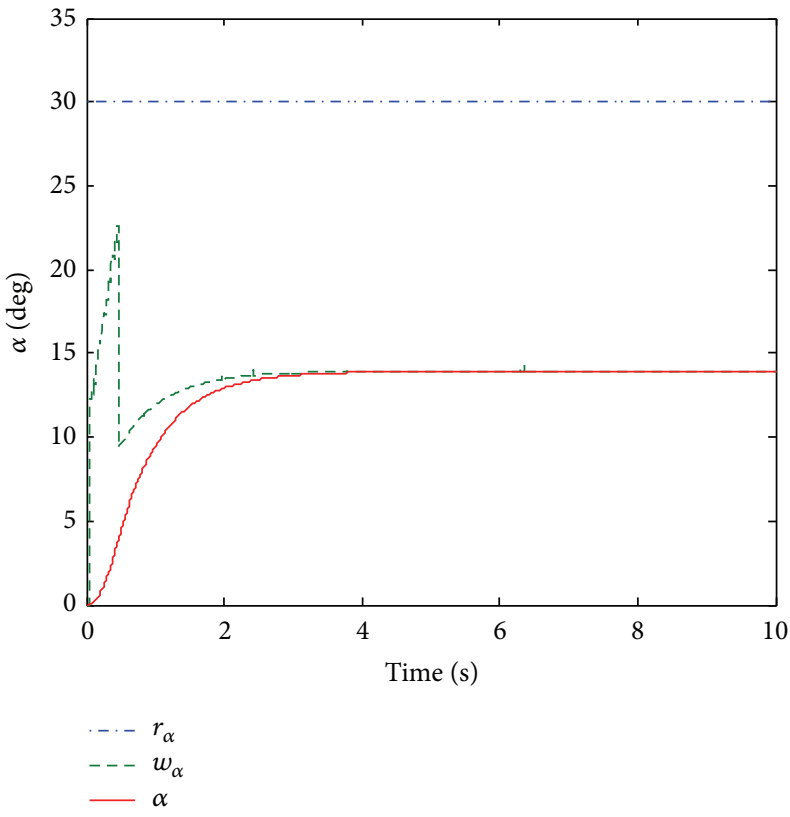

(b)

FIGURE 7: Simulation results of the static reference governor with state uncertainty set.

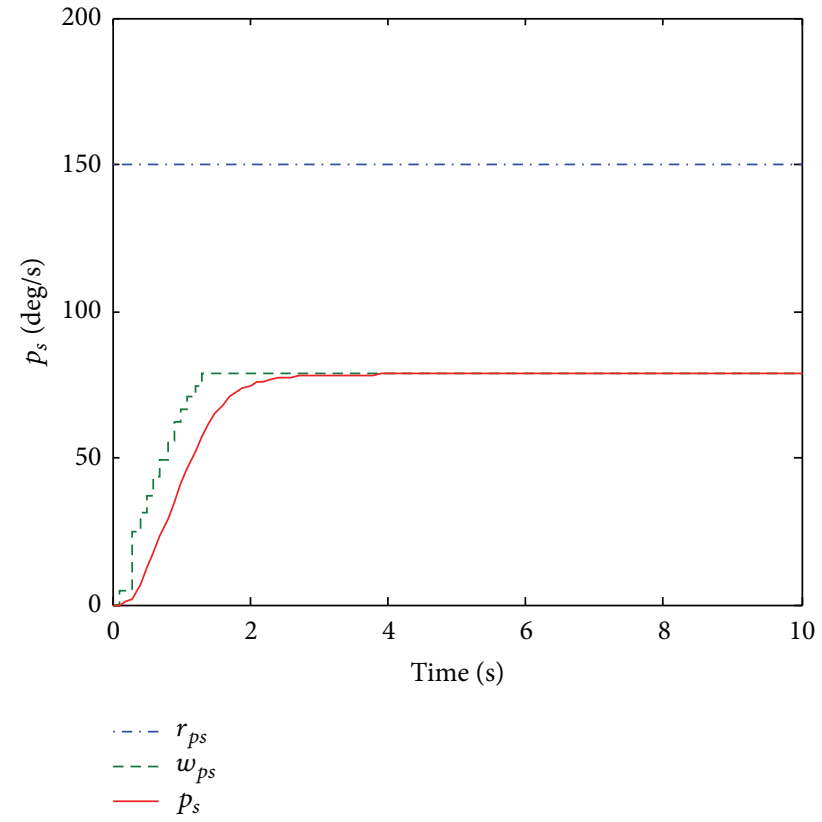

(a)

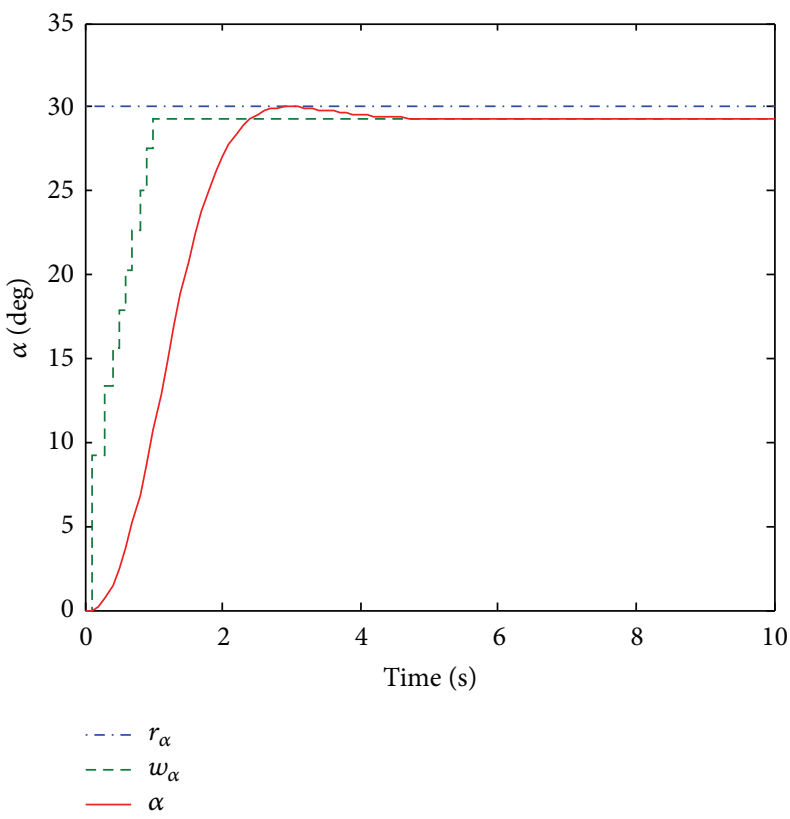

(b)

FIGURE 8: Simulation results of the dynamic reference governor with state uncertainty set.

6.1. Applications of Static and Dynamic Reference Governor. In this subsection, the static and the dynamic reference governors are employed to the flight envelope protection to handle the constraints imposed on input and output of the aircraft system. Because the nominal control law is designed based on continuous-time linear plant of the aircraft, the closed-loop system should be discretized to the form of (1) for the design of the reference governor with discrete type. The nominal control law (3) does not change in the discretization. Different sampling periods are used for static and dynamic reference governors, respectively. Zero-order holds are used at each output of the reference governor.

For the static reference governor, the system is discretized using the 0.01 second sampling period. The maximal admissible set is calculated by utilizing the method in Section 5.3. Without setting tolerance, a polyhedron in $R^{6}$ with 168 


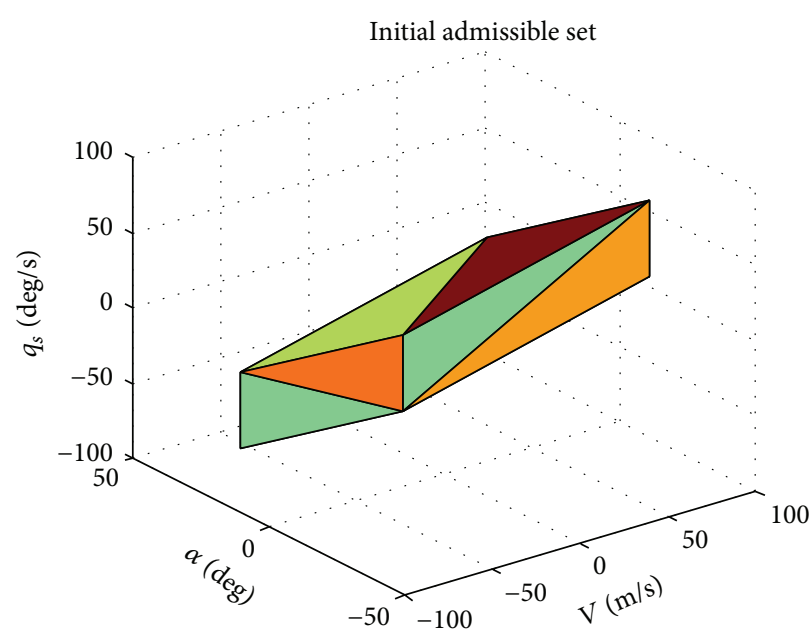

(a)

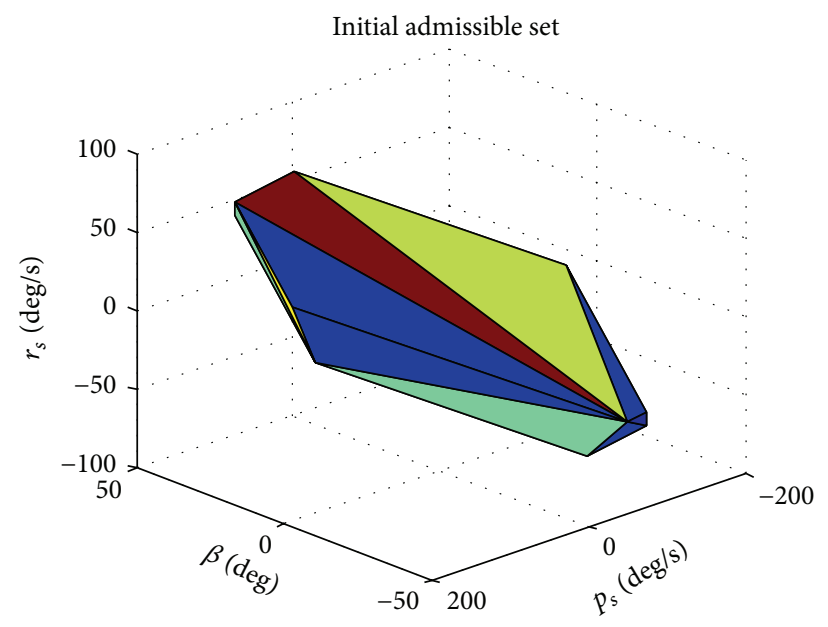

(b)

FIGURE 9: The initial admissible set of the inner closed-loop system.

inequality constraints is obtained after 52 iterations. The polyhedron is of more than three dimensions and is difficult to be displayed visually. Different profiles are produced when displayed in various three-dimensional coordinates. Figure 3 shows the profiles of the polyhedron in $V-\alpha-q_{s}$ and $\beta-p_{s}-r_{s}$ coordinates. Compared with the initial admissible set $\mathscr{X}^{0}$ decided by the input and output constraints, the profile in $V-\alpha-q_{s}$ coordinate does not change a lot. However, the profile in $\beta-p_{s}-r_{s}$ coordinates is largely trimmed.

The previously given step command in Section 5.3 is specified as the original reference command and the output of the reference governor and the closed-loop system is shown in Figure 4. It can be seen that the static reference governor prevents the deflection of control surfaces out of their limits successfully at the beginning of the simulation. However, the steady states of $p_{s}$ and $\alpha$ are far away from their commands while the control effectors still have margin by comparing with their saturation conditions. It is apparent that there exists an inherent drawback of the static reference governor.

The sampling period used in the discretization of the system for the dynamic reference governor is 0.1 seconds and the tolerance in the maximal admissible set calculation is set to be 0.000001 . Large sampling period and tolerance are selected to reduce the calculated quantity of the maximal admissible set. Additional constraints are imposed on the state of the dynamic reference governor $\left(w_{p s}, w_{\alpha}, w_{\beta}\right)^{T}$ as $w_{p s} \leq 150$, $w_{\alpha} \leq 30$, and $w_{\beta} \leq 30$. After 884 iterations, a polyhedron in $R^{9}$ with 2948 inequalities constraints is obtained, as shown in Figure 5. The profiles of the polyhedron in $V-\alpha-q_{s}$ and $\beta-p_{s}-r_{s}$ coordinates are same as in the static reference governor and the rough surfaces are caused by large tolerance.

Figure 6 shows the response of the system with the dynamic reference governor. In Figure 6, it is shown that the original step references $r_{p s}$ and $r_{\alpha}$ are modified through the reference governor and virtual signals $w_{p s}$ and $w_{\alpha}$ are generated. Tracking these signals, constraint violations of $\alpha$ and the deflection of control surfaces are avoided. When $\alpha$ reaches the upper limit, $p_{s}$ is about 128 degrees per second. This signal is considered as the nearest signal by $\mathbf{r}$ in the sense of 1-norm.

Simulations are also performed in the situation that $(V, \alpha, \beta)$ cannot be measured accurately, whereas $\left(p_{s}, q_{s}, r_{s}\right)$ are exactly known. The noises of $(V, \alpha, \beta)$ satisfy $|\xi(t)|<1$, $\forall t \in Z^{+}$. Reference governors with state uncertainty sets are employed. Simulation results of the static reference governor (21) and the dynamic reference governor (22) are showed in Figures 7 and 8, respectively. It can be seen that more conservative reference signals are generated due to the existence of the noise.

6.2. Application of Reference Governor Based on Step Response Model. The settling time $T_{s}$ of the continuous-time inner closed-loop system is determined after the nominal control law is given. The order of the step response model $N$ is then decided by the sampling period $\tau$ as $N=T_{s} / \tau$. In the application for flight envelope protection, we choose $T_{s}=4 \mathrm{~s}$ and $\tau=0.1 \mathrm{~s}$. Thus, we have $N=40$. Consequently, a 240thorder model is created in the simulation. Although the model is complex, iterative computations of the maximal admissible set are avoided when this model is applied for the reference governor. By contrast, it uses the initial admissible set which relies on the reference value at $t-1$. A typical initial admissible set $($ when $\mathbf{w}=0)$ is shown in Figure 9.

Simulation results of the system with the reference governor based on step response model are shown in Figure 10. It is shown that equally good properties as the dynamic reference governor can be achieved. However, the complex calculation of the maximal admissible set is not needed under this case.

From Figures 6 and 10, the dynamic reference governor and the reference governor based on step response model are valid in the flight envelope protection control.

\section{Conclusion}

In this paper, the reference governor strategy has been developed for the flight envelope protection control during 


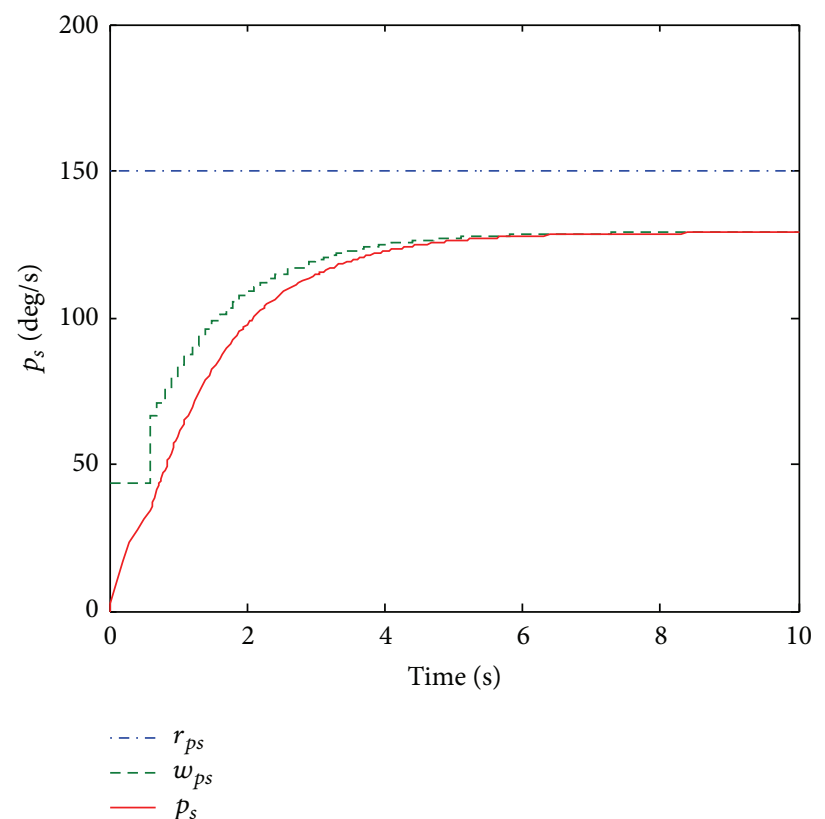

(a)

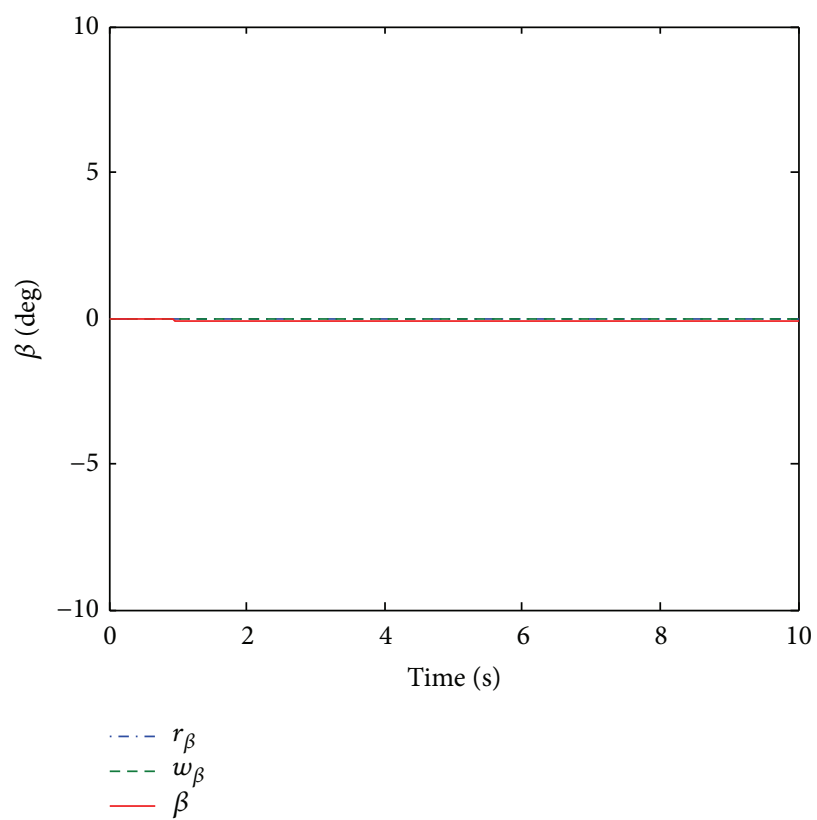

(c)

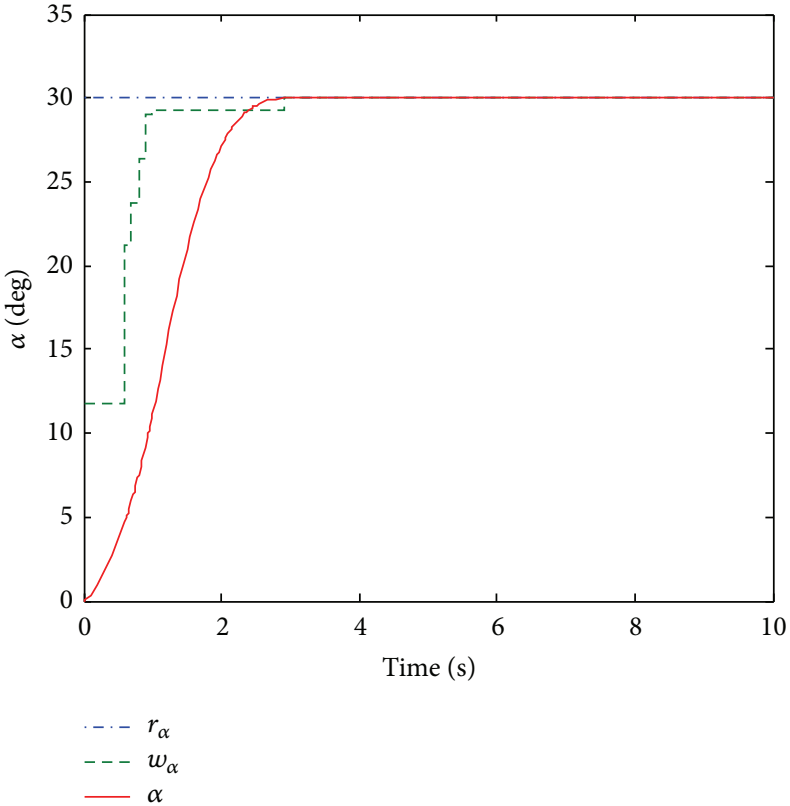

(b)

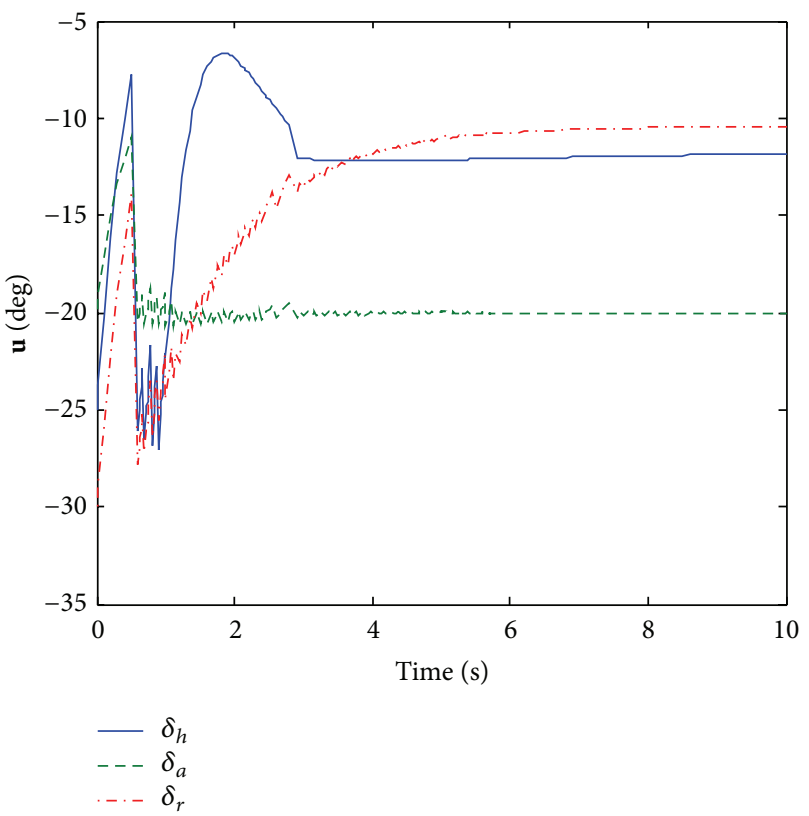

(d)

FIGURE 10: Simulation results of the reference governor based on step response model.

high angle of attack maneuvers. According to the simulation results, we can conclude the following: (i) the static reference governor has great conservatism on the admissible input and cannot be used for the flight envelope protection; (ii) the dynamic reference governor exhibits good properties to prevent constraint violation of key flight parameters and deflections of control surface, but the maximal admissible set is difficult to obtain and it has complex profile; (iii) the reference governor based on step response model proposed in Section 4 has good properties as well but does not need to calculate the complex maximal admissible set. When highdimensional models are utilized, more effective optimization algorithm should be developed for the large-scale optimization problem. This will be discussed in our future works.

\section{Conflict of Interests}

The authors declare that there is no conflict of interests regarding the publication of this paper. 


\section{Acknowledgments}

This research is supported by Jiangsu Natural Science Foundation of China (Grant no. SBK20130033), National Natural Science Foundation of China (Grant no. 61374212), and the NUAA Fundamental Research Funds (NS2013028).

\section{References}

[1] S. Tarbouriech, G. Garcia, and A. H. Glattfelder, Advanced Strategies in Control Systems with Input and Output Constraints, Springer, Berlin, Germany, 2006.

[2] M. Chen, Q. Wu, C. Jiang, and B. Jiang, "Guaranteed transient performance based control with input saturation for near space vehicles," Science China Information Sciences, vol. 57, no. 5, pp. 1-12, 2014.

[3] M. Chen, B. Jiang, Q. Wu, and C. Jiang, "Robust control of nearspace vehicles with input backlash-like hysteresis," Proceedings of the Institution of Mechanical Engineers. Part I: Journal of Systems and Control Engineering, vol. 227, no. 8, pp. 635-644, 2013.

[4] M. V. Kothare, V. Balakrishnan, and M. Morari, "Robust constrained model predictive control using linear matrix inequalities," Automatica, vol. 32, no. 10, pp. 1361-1379, 1996.

[5] D. Q. Mayne, J. B. Rawlings, C. V. Rao, and P. O. Scokaert, "Constrained model predictive control: stability and optimality," Automatica, vol. 36, no. 6, pp. 789-814, 2000.

[6] E. G. Gilbert, I. Kolmanovsky, and K. T. Tan, "Discrete-time reference governors and the nonlinear control of systems with state and control constraints," International Journal of Robust and Nonlinear Control, vol. 5, no. 5, pp. 487-504, 1995.

[7] E. G. Gilbert and I. Kolmanovsky, "Fast reference governors for systems with state and control constraints and disturbance inputs," International Journal of Robust and Nonlinear Control, vol. 9, no. 15, pp. 1117-1141, 1999.

[8] A. Bemporad, A. Casavola, and E. Mosca, "Nonlinear control of constrained linear systems via predictive reference management," IEEE Transactions on Automatic Control, vol. 42, no. 3, pp. 340-349, 1997.

[9] A. Casavola, E. Mosca, and D. Angeli, "Robust command governors for constrained linear systems," IEEE Transactions on Automatic Control, vol. 45, no. 11, pp. 2071-2077, 2000.

[10] A. Casavola, E. Mosca, and M. Papini, "Control under constraints: an application of the command governor approach to an inverted pendulum," IEEE Transactions on Control Systems Technology, vol. 12, no. 1, pp. 193-204, 2004.

[11] K. Kogiso and K. Hirata, "Reference governor for constrained systems with time-varying references," Robotics and Autonomous Systems, vol. 57, no. 3, pp. 289-295, 2009.

[12] D. Angeli and E. Mosca, "Command governors for constrained nonlinear systems," IEEE Transactions on Automatic Control, vol. 44, no. 4, pp. 816-820, 1999.

[13] E. G. Gilbert and I. V. Kolmanovsky, "Set-point control of nonlinear systems with state and control constraints: a Lyapunovfunction, reference-governor approach," in Proceedings of the 38th IEEE Conference on Decision and Control (CDC '99), pp. 2507-2512, December 1999.

[14] E. Gilbert and I. Kolmanovsky, "Nonlinear tracking control in the presence of state and control constraints: a generalized reference governor," Automatica, vol. 38, no. 12, pp. 2063-2073, 2002.
[15] M. Pachter and P. R. Chandler, "Velocity vector roll control," in Proceedings of the AIAA, Guidance, Navigation and Control Conference, AIAA Paper 96-3867, 1996.

[16] J.-S. Yee, J. L. Wang, and N. Sundararajan, "Robust sampleddata $H_{\infty}$-flight-controller design for high $\alpha$ stability-axis roll maneuver," Control Engineering Practice, vol. 8, no. 7, pp. 735$747,2000$.

[17] E. R. van Oort, Q. P. Chu, J. A. Mulder, and T. J. J. van Den Boom, "Robust model predictive control of a feedback linearized nonlinear F-16/MATV aircraft model," in Proceedings of the AIAA Guidance, Navigation, and Control Conference and Exhibit, pp. 2148-2174, AIAA, August 2006.

[18] G. Grimm, J. Hatfield, I. Postlethwaite, A. R. Teel, M. C. Turner, and L. Zaccarian, "Antiwindup for stable linear systems with input saturation: an LMI-based synthesis," IEEE Transactions on Automatic Control, vol. 48, no. 9, pp. 1509-1525, 2003.

[19] W. Falkena, C. Borst, Q. P. Chu, and J. A. Mulder, "Investigation of practical flight envelope protection systems for small aircraft," Journal of Guidance, Control, and Dynamics, vol. 34, no. 4, pp. 976-988, 2011.

[20] F. Blanchini and S. Miani, Set-Theoretic Methods in Control, Springer, 2007.

[21] S. Miani and C. Savorgnan, "MAXIS-G: a software package for computing polyhedral invariant sets for constrained LPV systems," in Proceedings of the 44th IEEE Conference on Decision and Control, and the European Control Conference (CDC-ECC '05), pp. 7609-7614, December 2005.

[22] D. Angeli, A. Casavola, and E. Mosca, "On feasible set-membership state estimators in constrained command governor control," Automatica, vol. 37, no. 1, pp. 151-156, 2001.

[23] H. Chen, Model Predictive Control, Science Press, Beijing, China, 2013.

[24] Standard Military, "Flying qualities of piloted vehicles," Tech. Rep. MIL-STD-1797A, US Air Force Flight Dynamics Laboratory, 1990.

[25] W. C. Reigelsperger, S. S. Banda, and D. P. Lemaster, "Application of multivariable control theory to aircraft control laws," WL-TR 96-3099, Flight Dynamics Directorate, WrightPatterson Air Force Base, 1996. 


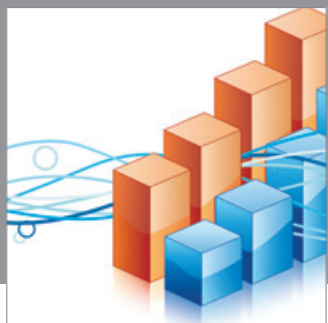

Advances in

Operations Research

mansans

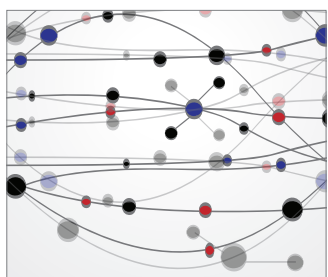

The Scientific World Journal
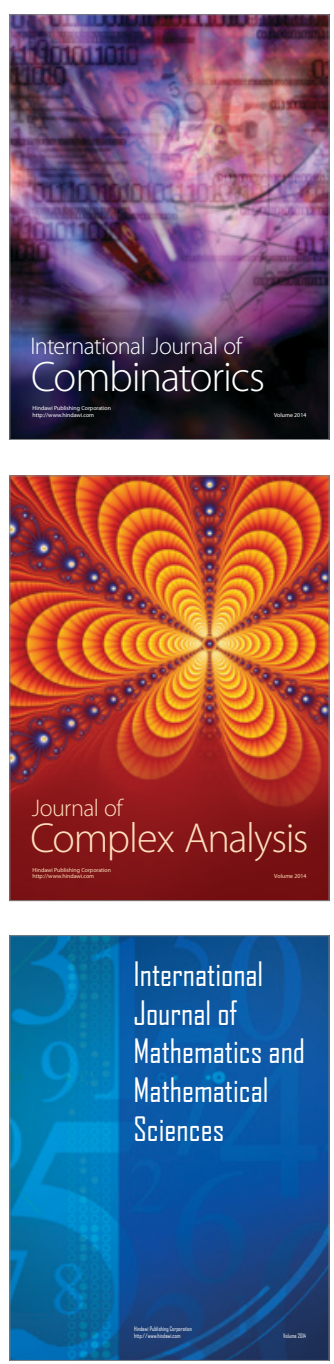
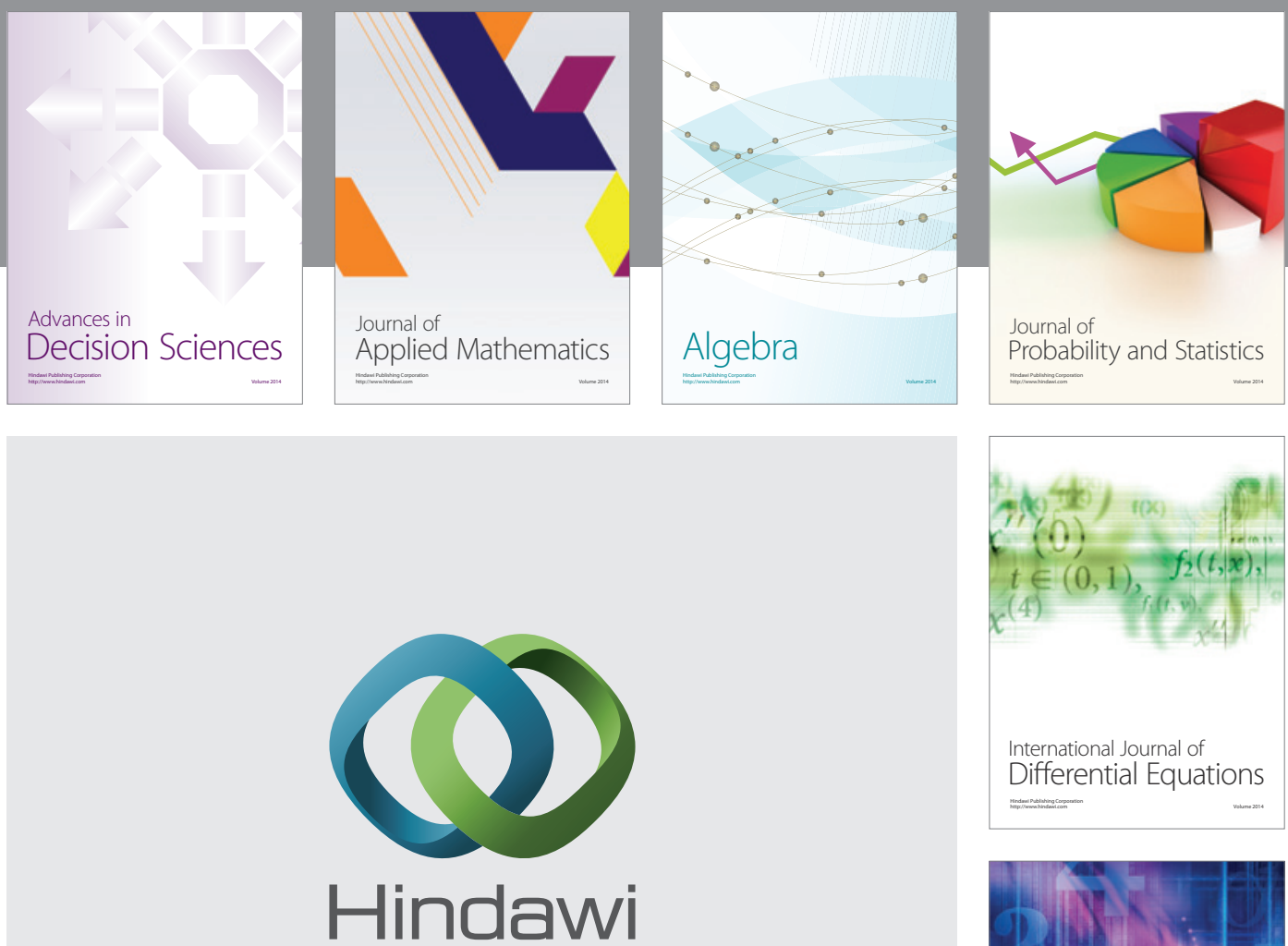

Submit your manuscripts at http://www.hindawi.com
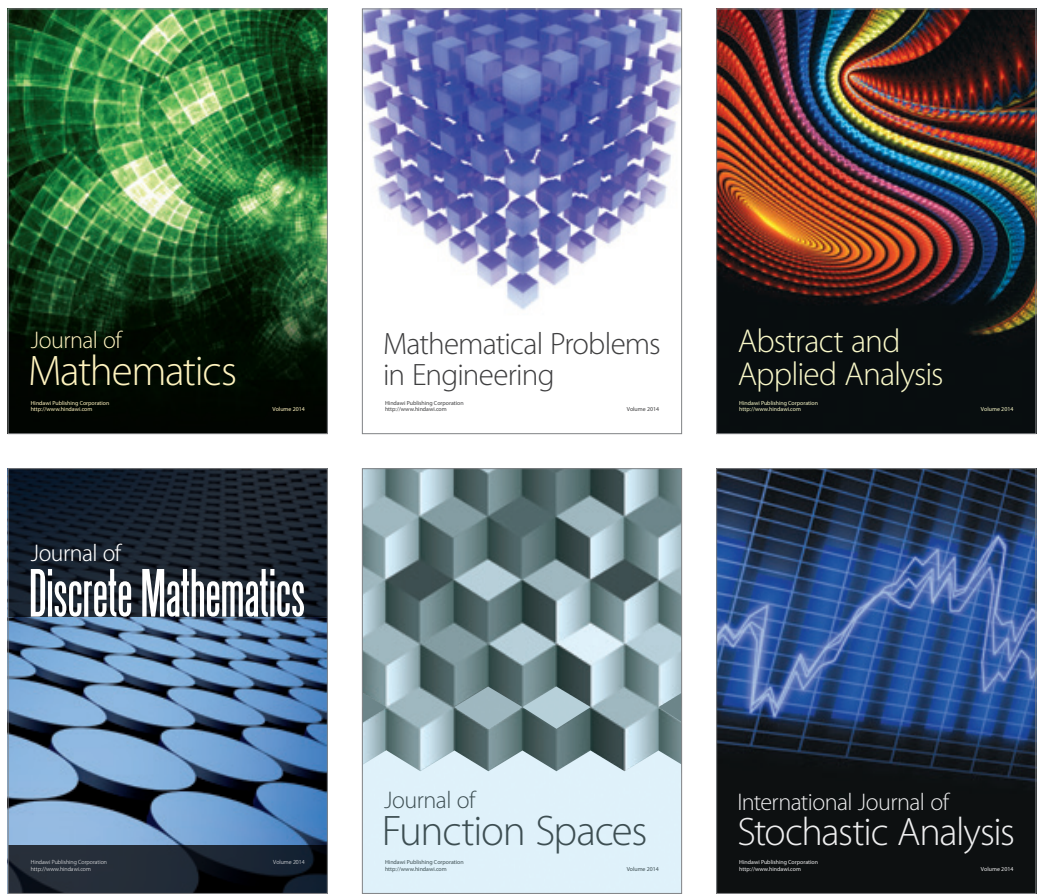

Journal of

Function Spaces

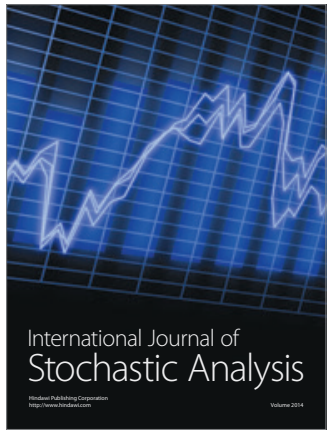

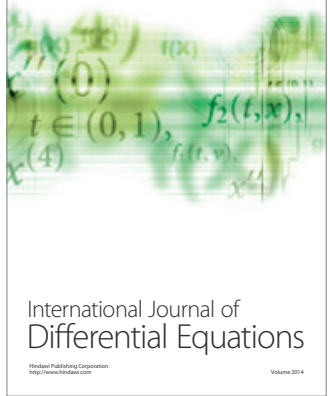
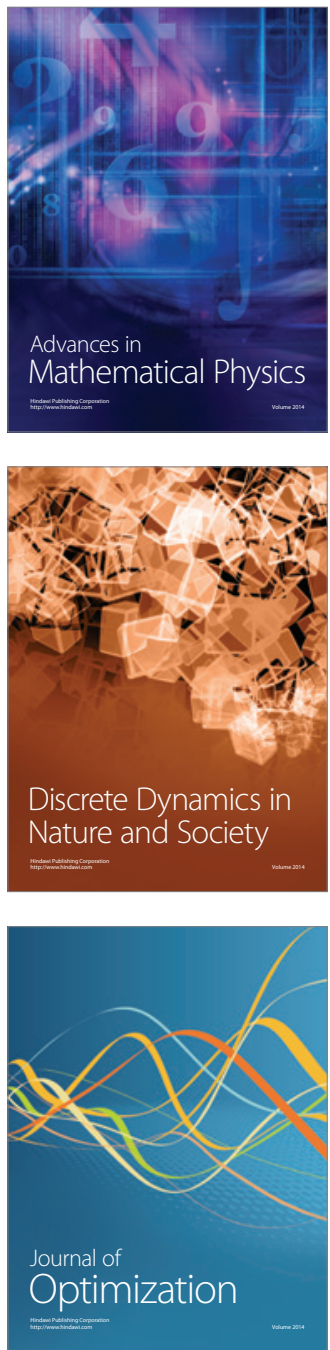